\title{
Spectrum and Analytic Functional Calculus for Clifford Operators via Stem Functions
}

https://doi.org/10.1515/conop-2020-0115

Received January 25, 2021; accepted May 25, 2021

Abstract: The main purpose of this work is the construction of an analytic functional calculus for Clifford operators, which are operators acting on certain modules over Clifford algebras. Unlike in some preceding works by other authors, we use a spectrum defined in the complex plane, and also certain stem functions, analytic in neighborhoods of such a spectrum. The replacement of the slice regular functions, having values in a Clifford algebra, by analytic stem functions becomes possible because of an isomorphism induced by a Cauchy type transform, whose existence is proved in the first part of this work.

Keywords: Clifford algebras; stem functions; analytic functional calculus; Clifford and complex spectra

MSC: 30G35; 30A05; 47A10; 47A60

\section{Introduction}

It is unanimously admitted that the analytic functional calculus is a basic tool in the study of linear operators. While the case of a single operator is settled by the Riesz-Dunford functional calculus (see for instance [4]), the case of several operators is more complicate even in the case of commuting ones, because of the non canonical character of Cauchy type formulas analytic functions in several variables, defined in neighborhoods of joint spectra. Nevertheless, a complete construction for the case of commuting tuples of Banach space operators can be found in the papers [14,15], and which was applied, in particular, in investigations related to the local spectral theory (see $[5,17]$ etc.).

The case of not necessarily commuting tuples of operators has been approached by several authors, whose important contributions can be found in works like [12,16], and also in [2,10], where significant results are obtained by associating the tuples of operators with some Clifford algebras, which reduces the discussion to the case of a single operator.

In the present paper, we also associate the tuples of operators with some Clifford algebras but, unlike in $[2,3,10]$, we replace the slice regular functions (see Subsection 2.2), defined in some open subsets of Clifford algebras, by analytic stem functions (see Definition 3), defined in the complex plane. This is a consquence of adopting a different concept of spectrum, that from [2] being defined as a subset of a Clifford algebra, identified with an Euclidean space, while the spectrum in this work is defined in the complex plane. Nevertheless, the analytic functional calculi, obtained in two ways, are shown to be equivalent (see Remark 17). This equivalence is based on the isomorphism of the corresponding spaces of functions which are used in these constructions, and it is a consequence of some results proved in the first part of this work (see our Theorem 5).

As in the case of Hamilton's algebra of quaternions, an important investigation in the context of Clifford algebras has been to find a convenient manner to express the "analyticity" of functions, defined on subsets of such algebras.

^Corresponding Author: Florian-Horia Vasilescu: Department of Mathematics, University of Lille, 59655 Villeneuve d'Ascq, France, E-mail: florian.vasilescu@univ-lille.fr 
A concept of S-monogenic function was introduced in [1], generalizing that of slice regularity (see [7]) to the framework of Clifford algebres, leading to a significant development sythesized in the first part of [2] (which contains a large list of references), whose impact is still actual.

Unlike in [1], the basic idea of the present paper is to define the regularity of functions, taking values in a Clifford algebra, via an analytic functional calculus, adapting the corresponding results in the Hamilton algebra context from [18].

Roughly speaking, and unlike in [7], an " $S$-monogenic function" (which, for simplicity, will be often called in this text "slice regular") can and will be obtained by a pointwise application of the analytic functional calculus with stem functions on a conjugate symmetric open set $U$ in the complex plane, to some elements of a given Clifford algebra called paravectors (see Subsection 2.1), whose spectra are in $U$, via the matrix version of Cauchy's formula (11), with no need of slice derivatives. In this way, we obtain a whole class of "regular functions" (in fact, Cauchy transforms of stem functions), eventually shown that this is precisely the class of $S$-monogenic functions, by Theorem 5.

Then we initiate some elementary spectral theory for what we call Clifford operators (defined in Subsection 2.3), acting on two-sided modules over a Clifford algebra. A functional calculus with analytic functions is also presented, which happens to be equivalent to the calculus with $S$-monogenic functions, as already mentioned above.

The advantage of our approach is its simplicity, and its connection with the classical theory, using a spectrum in the complex plane and a Riesz-Dunford type of a kernel.

The structure of this work is the following. Besides this introduction, there is a preliminary section, presenting mostly well known concepts and result, necessary in the sequel. The rest of the work may be virtually divided into two parts. A first part (including Sections 3-6) deals with a description of an analytic functional calculus for stem functions, while the second part (including Sections 7-8) presents elements of spectral theory for Clifford operators.

This work is inspired by the author's articles $[18,19]$, dedicated to the quaternionic case, several results having similar proofs to the corresponding one from the quoted papers, via minor modifications. Nevertheless, for the sake of completeness, and for the convenience of the readrs, we often give full arguments. Some fairly new results are presented in Corollary 2, where the so-called intrinsic functions (see [2], Definition 3.5.1 of [3], Definition 2.1.2) are described in terms of complex-valued stem functions, and in Remark 18, giving a version of the spectral mapping theorem, which, unlike in [2], Theorem 3.5.9, appears in a classical framework.

\section{Preliminaries}

\subsection{Clifford Algebra and its Complexification}

First of all, we introduce the concept of (real) Clifford algebra, in a restricted sense (as in [2]; see also [9, 10, 13] etc.). Specifically, in this text, by Clifford algebra, denoted by $\mathfrak{C}_{n}$ for a fixed integer $n \geq 0$, we mean the unital associative real algebra having $n+1$ generators, say $e_{0}=1, e_{1}, \ldots, e_{n}$, satisfying the relations $e_{j}^{2}=-1$, and $e_{j} e_{k}=-e_{k} e_{j}$ for all $j, k=1, \ldots, n, j \neq k$. In particular, the real algebra $\mathbb{R}$ is a subalgebra of $\mathfrak{C}_{n}$. In fact, $\mathfrak{C}_{0}=\mathbb{R}, \mathfrak{C}_{1}=\mathbb{C}$, and $\mathfrak{C}_{2}=\mathbb{H}$, that is, the the real, complex and quaternionic algebras are special cases of Clifford algebras.

Setting $\mathbb{N}_{n}=\{1,2, \ldots, n\}$, for every ordered subset $J=\left\{j_{1}, j_{2}, \cdots, j_{p}\right\} \subset \mathbb{N}_{n}$, with $j_{1}<j_{2}<\cdots<j_{p}$ and $1 \leq p \leq n$, we put $e_{J}=e_{j_{1}} e_{j_{2}} \cdots e_{j_{p}}$. We use the symbol $J \prec \mathbb{N}_{n}$ to indicate that $J$ is an oredered set as above. We also assume that $\emptyset \prec \mathbb{N}_{n}, e_{\emptyset}=1, e_{\{j\}}=e_{j}, j=1, \ldots, n$, and that the family $\left\{e_{J}\right\}_{J \prec \mathbb{N}_{n}}$ is a basis of the vector space $\mathfrak{C}_{n}$. Therefore, an arbitrary element $\mathbf{a} \in \mathfrak{C}_{n}$ can be written as

$$
\mathbf{a}=\sum_{J \prec \mathbb{N}_{n}} a_{J} e_{J},
$$


where $a_{J} \in \mathbb{R}$ are uniquely determined for all $J \prec \mathbb{N}_{n}$. To simplify the notation, we shall put $e_{0}=e_{\emptyset}=1$, and $a_{0}=a_{\emptyset}$. The elements of the Clifford algebra $\mathfrak{C}_{n}$ will be sometimes called Clifford vectors, or briefly, Cl-vectors.

The linear subspace of $\mathfrak{C}_{n}$ spanned by $\left\{\boldsymbol{e}_{j}\right\}_{j=0}^{n}$ will be denoted by $\mathfrak{P}_{n}$. It plays an important role throughout this work. The $\mathrm{Cl}$-vectors from the subspace $\mathfrak{P}_{n}$, which have the form $\mathbf{a}=a_{0}+\sum_{k=0}^{n} a_{k} e_{k}$ with $a_{k} \in \mathbb{R}$ for all $k=0, \ldots, n$, will be called paravectors (as in [2]). The linear subspace $\mathfrak{P}_{n}$ will be often identified with the Euclidean space $\mathbb{R}^{n+1}$, via the linear isomorphism

$$
\mathfrak{P}_{n} \ni \sum_{k=0}^{n} a_{k} e_{k} \mapsto\left(a_{0}, a_{1}, \ldots, a_{n}\right) \in \mathbb{R}^{n+1} .
$$

For every $\mathbf{a}=\sum_{J \prec \mathbb{N}_{n}} a_{J} e_{J} \in \mathfrak{C}_{n}$ we have a decomposition $\mathbf{a}=\Re(\mathbf{a})+\Im(\mathbf{a})$, where $\Re(\mathbf{a})=a_{0}$ and $\Im(\mathbf{a})=$ $\sum_{\emptyset \neq J \prec \mathbb{N}_{n}} a_{J} e_{J}$, that is, the real part and the imaginary part of the $\mathrm{Cl}$-vector $\mathbf{a} \in \mathfrak{C}_{n}$, respectively.

The algebra $\mathfrak{C}_{n}$ has a norm defined by

$$
|\mathbf{a}|^{2}=\sum_{J \prec \mathbb{N}_{n}} a_{J}^{2},
$$

where $\mathbf{a}$ is given by (1).

The algebra $\mathfrak{C}_{n}$ also has an involution $\mathfrak{C}_{n} \ni \mathbf{a} \mapsto \mathbf{a}^{\star} \in \mathfrak{C}_{n}$, which is defined via the conditions $e_{j}^{\star}=-e_{j}(j=$ $1, \ldots, n), r^{\star}=r \in \mathbb{R}$, and $(\mathbf{a b})^{\star}=\mathbf{b}^{\star} \mathbf{a}^{\star}$ for all $\mathbf{a}, \mathbf{b} \in \mathfrak{C}_{n}$ (see [2], Definition 2.1.11). According to Proposition 2.1.12 from [2], we therefore have $\left(\mathbf{a}^{\star}\right)^{\star}=\mathbf{a}$, and $(\mathbf{a}+\mathbf{b})^{\star}=\mathbf{a}^{\star}+\mathbf{b}^{\star}$. Particularly, if $\mathbf{a}=a_{0}+\sum_{j=1}^{n} a_{j} e_{j}$, then $\mathbf{a}^{\star}=a_{0}-\sum_{j=1}^{n} a_{j} e_{j}$, for all $\mathbf{a} \in \mathfrak{P}_{n}$.

Remark 1. Unlike in [2], we keep the name of conjugation for a different concept (as in [18], for the case of quaternions).

To define the conjugation, we consider the complexification $\mathfrak{K}_{n}=\mathbb{C} \otimes_{\mathbb{R}} \mathfrak{C}_{n}$, identified with the direct sum $\mathfrak{C}_{n}+i \mathfrak{C}_{n}$, and so $\mathfrak{K}_{n} \supset \mathbb{C}$. This is a unital algebra with the involution induced by the involution of $\mathfrak{C}_{n}$ :

$$
\mathfrak{K}_{n} \ni \mathbf{c}=\mathbf{a}+i \mathbf{b} \mapsto \mathbf{c}^{\star}=\mathbf{a}^{\star}-i \mathbf{b}^{\star} \in \mathfrak{K}_{n}
$$

for all $\mathbf{a}, \mathbf{b} \in \mathfrak{C}_{n}$.

The $\mathbb{R}$-linear map

$$
\mathfrak{K}_{n} \ni \mathbf{c}=\mathbf{a}+i \mathbf{b} \mapsto \overline{\mathbf{c}}:=\mathbf{a}-i \mathbf{b} \in \mathfrak{K}_{n}
$$

is a conjugation on $\mathfrak{K}_{n}$, that is, a real automorphism of a unital algebra, whose square is the identity.

An important feature of this construction is that the elements of the real subalgebra $\mathfrak{C}_{n}$ commute with the complex numbers in the algebra $\mathfrak{K}_{n}$. Of course, $\overline{\mathbf{a}}=\mathbf{a}$ if and only if $\mathbf{a} \in \mathfrak{C}_{n}$, which is useful criterion to identify the elements of $\mathfrak{C}_{n}$ among those of $\mathfrak{K}_{n}$.

\subsection{Slice Regular Functions}

The subspace $\mathfrak{P}_{n}$ of paravectors in a Clifford algebra $\mathfrak{C}_{n}$ will play an important role in what follows. In fact, we are particularly interested in functions defined on open subsets of $\mathfrak{P}_{n}$ (which is identified with $\mathbb{R}^{n+1}$ ), with values in $\mathfrak{C}_{n}$.

We shall be dealing with the concept of "slice regularity" of such functions, which is a form of holomorphy (introduced in [1]; see also [2] and several works quoted within).

If $\mathbf{a}=a_{0}+\sum_{k=1}^{n} a_{k} e_{k} \in \mathfrak{P}_{n}$ is arbitrary, and so $\mathbf{a}^{\star}=a_{0}-\sum_{k=1}^{n} a_{k} e_{k} \in \mathfrak{P}_{n}$, we have

$$
\mathbf{a a}^{\star}=\mathbf{a}^{\star} \mathbf{a}=\sum_{k=0}^{n} a_{k}^{2}=|\mathbf{a}|^{2} .
$$

This shows that the nonnull paravectors $\mathbf{a} \in \mathfrak{P}_{n}$ are invertible in the algebra $\mathfrak{C}_{n}$ and, in fact, $\mathbf{a}^{-1}=$ $|\mathbf{a}|^{-2} \mathbf{a}^{\star} \in \mathfrak{P}_{n}$. 
For $\mathfrak{K}_{n}$-valued functions defined on subsets of $\mathfrak{P}_{n}$, the concept of slice regularity (see also [2]) is defined as follows.

Let $\mathbb{S}_{n}=\left\{\mathfrak{s}=\sum_{k=1}^{n} s_{k} e_{k} ; \sum_{k=1}^{n} s_{k}^{2}=1\right\}$, that is, the unit sphere of purely imaginary elements of $\mathfrak{P}_{n}$. It is clear that $\mathfrak{s}^{\star}=-\mathfrak{s}, \mathfrak{s}^{2}=-1, \mathfrak{s}^{-1}=-\mathfrak{s}$, and $|\mathfrak{s}|=1$ for all $\mathfrak{s} \in \mathbb{S}_{n}$. Moreover, every nonnull paravector a can be written as $\mathbf{a}=\Re(\mathbf{a})+|\Im(\mathbf{a})| \mathfrak{s}_{\mathbf{a}}$, with $\mathfrak{s}_{\mathbf{a}}=|\Im(a)|^{-1} \Im(\mathbf{a}) \in \mathbb{S}_{n}$ if $\Im(\mathbf{a}) \neq 0$, and $\mathfrak{s}_{\mathbf{a}}=e_{1}$ otherwise.

Now, let $\Omega \subset \mathfrak{P}_{n}$ be an open set, and let $F: \Omega \mapsto \mathfrak{K}_{n}$ be a differentiable function. In the spirit of [1, 2], we say that $F$ is right slice regular on $\Omega$ if for all $\mathfrak{s} \in \mathbb{S}_{n}$,

$$
\bar{\partial}_{\mathfrak{s}} F(x+y \mathfrak{s}):=\frac{1}{2}\left(\frac{\partial}{\partial x}+R_{\mathfrak{s}} \frac{\partial}{\partial y}\right) F(x+y \mathfrak{s})=0,
$$

on the set $\Omega \cap(\mathbb{R}+\mathbb{R} \mathfrak{s})$, where $R_{\mathfrak{s}}$ is the right multiplication of the elements of $\mathfrak{K}_{n}$ by $\mathfrak{s}$.

Unlike in [2], we use the right slice regularity rather than the left one because of a reason to be later explained (see Remark 8(1)). Nevertheless, a left slice regularity can also be defined via the left multiplication of the elements of $\mathfrak{K}_{n}$ by elements from $\mathbb{S}_{n}$. In what follows, the right slice regularity will be simply called slice regularity.

As mentioned before, we are particularly interested by the slice regularity of $\mathfrak{C}_{n}$-valued functions, but the concept is valid for $\mathfrak{K}_{n}$-valued functions and plays an important role in our discussion.

Example 1. (1) The convergent series of the form $\sum_{m \geq 0} \mathbf{a}_{m} \kappa^{m}$, on balls $\left\{\kappa \in \mathfrak{P}_{n} ;|\kappa|<r\right\}$, with $r>0$ and $\mathbf{a}_{m} \in \mathfrak{K}_{n}$ for all $m \geq 0$, are $\mathfrak{K}_{n}$-valued slice regular on their domain of definition. Of course, when $\mathbf{a}_{m} \in \mathfrak{C}_{n}$ for all $m$, such functions are $\mathfrak{C}_{n}$-valued slice regular on their domain of definition.

Remark 2. The previous discussion is somehow unseemly when considering the quaternionic algebra $\mathbb{H}=$ $\mathfrak{C}_{2}$. Indeed, in this case we are mainly interested in $\mathbb{H}$-valued functions, defined on subsets of $\mathbb{H}$. The algebra $\mathfrak{C}_{2}$ is generated by $\left\{1, e_{1}, e_{2}\right\}$, and the vector space $\mathfrak{P}_{2}$ generated by this set is strictly included in $\mathbb{H}$. As the algebra $\mathbb{H}$ is also generated by the set $\left\{1, e_{1}, e_{2}, e_{3}\right\}$, where $e_{3}=e_{1} e_{2}$, it is isomorphic to the quotient of the algebra $\mathfrak{C}_{3}$ by the two-sided ideal generated by $e_{3}-e_{1} e_{2}$. Consequently, a separate approach concerning the quaternion algebra $\mathbb{H}$ (as in [2]), rather than an approach in the framework of Clifford algebras, seems to be more appropriate, because it is not a particular case (for $n=2$ ) of the present approach. In fact the case $\mathfrak{C}_{2}=\mathbb{H}$ is treated in the works $[18,19]$, strongly related to the present work.

Note also that for $\mathbb{C}=\mathfrak{C}_{1}$ we have $\mathfrak{P}_{1}=\mathbb{C}$.

\subsection{Clifford Spaces and Clifford Operators}

Roughly speaking, by a Clifford space (or a Cl-space) we mean a a two-sided module over a given Clifford algebra $\mathfrak{C}_{n}$. A Clifford space is, in particular, a real vector space. A more precise terminology will be given in the following.

Let $\mathfrak{C}_{n}$ be a fixed Clifford algebra, and let $\mathcal{V}$ be a real vector space. Adapting the framework from [2], the space $\mathcal{V}$ is said to be a right $\mathrm{Cl}$-space if it is a right $\mathfrak{C}_{n}$ - module, that is, there exists in $\mathcal{V}$ a right multiplication with the elements of $\mathfrak{C}_{n}$, such that $x 1=x,(x+y) \mathbf{a}=x \mathbf{a}+y \mathbf{a}, x(\mathbf{a}+\mathbf{b})=x \mathbf{a}+x \mathbf{b}, x(\mathbf{a b})=(x \mathbf{a}) \mathbf{b}$ for all $x, y \in \mathcal{V}$ and $\mathbf{a}, \mathbf{b} \in \mathfrak{C}_{n}$.

If $\mathcal{V}$ is a right $\mathrm{Cl}$-space which is also a Banach space with the norm $\left\|{ }^{\star}\right\|$ such that $\|x \mathbf{a}\| \leq C\|x\||\mathbf{a}|$ for all $x \in \mathcal{V}$ and $\mathbf{a} \in \mathfrak{C}_{n}$, where $C$ is a positive constant, then $\mathcal{V}$ is said to be a right Banch Cl-space.

In a similar way, one defines the concept of a left $\mathrm{Cl}$-space and that of a left Banach $\mathrm{Cl}$-space.

A real (Banach) vector space $\mathcal{V}$ will be said to be a (Banach) $\mathrm{Cl}$-space if it is simultaneously a right and a left (Banach) $\mathrm{Cl}$-space.

As for the case of quaternionic operators (see $[2,19]$ ), it seems to be the framework of Banch $\mathrm{Cl}$-spaces an appropriate one for the study of some specific linear operators, to be defined in the following.

If $\mathcal{V}$ is a real or complex Banach space, we denote by $\mathcal{B}(\mathcal{V})$ the algebra of all real or complex bounded linear operators, respectively. 
Let $\mathcal{V}$ be a fixed Banach $\mathrm{Cl}$-space. An operator $T \in \mathcal{B}(\mathcal{V})$ is said to be right Cl-linear if $T(x \mathbf{a})=T(x) \mathbf{a}$ for all $x \in \mathcal{V}$ and $\mathbf{a} \in \mathfrak{C}_{n}$. The set of right $C l$-linear operators will be denoted by $\mathcal{B}^{r}(\mathcal{V})$, which is, in particular, a unital real Banach algebra.

We shall denote by $R_{\mathbf{a}}$ (resp. $L_{\mathbf{a}}$ ) the right (resp. left) multiplication operator of the elements of $\mathcal{V}$ with the $\mathrm{Cl}$-vector $\mathbf{a} \in \mathfrak{C}_{n}$. It is clear that $R_{\mathbf{a}}, L_{\mathbf{a}} \in \mathcal{B}(\mathcal{V})$ for all $\mathbf{a} \in \mathfrak{C}_{n}$. Note also that

$$
\mathcal{B}^{\mathrm{r}}(V)=\left\{T \in \mathcal{B}(\mathcal{V}) ; T R_{\mathbf{a}}=R_{\mathbf{a}} T, \mathbf{a} \in \mathfrak{C}_{n}\right\} .
$$

The elements of the algebra $\mathcal{B}^{\mathrm{r}}(\mathcal{V})$ will be sometimes called right Clifford (or Cl-) operators. As we work especially with such operators, the word "right“ will be usually omitted. Note that all operators $L_{\mathbf{a}}, \mathbf{a} \in \mathfrak{C}_{n}$, are $\mathrm{Cl}$-operators.

Now, let us consider the complexification $\mathcal{V}_{\mathbb{C}}$ of $\mathcal{V}$, written as $\mathcal{V}_{\mathbb{C}}=\mathcal{V}+i \mathcal{V}$. Because $\mathcal{V}$ is a $\mathfrak{C}_{n}$-bimodule, the space $\mathcal{V}_{\mathbb{C}}$ is actually a two-sided $\mathfrak{K}_{n}$-module, via the multiplications

$$
(\mathbf{a}+i \mathbf{b})(x+i y)=\mathbf{a} x-\mathbf{b} y+i(\mathbf{a} y+\mathbf{b} x),(x+i y)(\mathbf{a}+i \mathbf{b})=x \mathbf{a}-y \mathbf{b}+i(y \mathbf{a}+x \mathbf{b}),
$$

for all $\mathbf{a}, \mathbf{b} \in \mathfrak{C}_{n}, x, y \in \mathcal{V}$.

For every $T \in \mathcal{B}(\mathcal{V})$, we consider its natural "complex extension“ to $\mathcal{V}_{\mathbb{C}}$ given by $T_{\mathbb{C}}(x+i y)=T x+i T y$, for all $x, y \in \mathcal{V}$, which is clearly $\mathbb{C}$-linear, so $T_{\mathbb{C}} \in \mathcal{B}\left(\mathcal{V}_{\mathbb{C}}\right)$. In fact, the map $\mathcal{B}(\mathcal{V}) \ni T \mapsto T_{\mathbb{C}} \in \mathcal{B}\left(\mathcal{V}_{\mathbb{C}}\right)$ is a unital injective morphism of real algebras. Moreover, if $T \in \mathcal{B}^{r}(\mathcal{V})$, the operator $T_{\mathbb{C}}$ is right $\mathfrak{K}_{n}$-linear, that is $T_{\mathbb{C}}((x+i y)(\mathbf{a}+i \mathbf{b}))=T_{\mathbb{C}}(x+i y)(\mathbf{a}+i \mathbf{b})$ for all $\mathbf{a}+i \mathbf{b} \in \mathfrak{K}_{n}, x+i y \in \mathcal{V}_{\mathbb{C}}$, via a direct computation.

The left and right multiplications with $\mathbf{a} \in \mathfrak{C}_{n}$ on $\mathcal{V}_{\mathbb{C}}$ will be still denoted by $L_{\mathbf{a}}, R_{\mathbf{a}}$, respectively, as elements of $\mathcal{B}\left(\mathcal{V}_{\mathbb{C}}\right)$. We set

$$
\mathcal{B}^{\mathrm{r}}\left(\mathcal{V}_{\mathbb{C}}\right)=\left\{S \in \mathcal{B}\left(\mathcal{V}_{\mathbb{C}}\right) ; S R_{\mathbf{a}}=R_{\mathbf{a}} S, \mathbf{a} \in \mathfrak{C}_{n}\right\},
$$

which is a unital complex algebra, consisting of all right $\mathfrak{K}_{n}$-linear operators on $\mathcal{V}_{\mathbb{C}}$, containing all operators $L_{\mathbf{a}}, \mathbf{a} \in \mathfrak{C}_{n}$. It is easily seen that if $T \in \mathcal{B}^{\mathrm{r}}(\mathcal{V})$, then $T_{\mathbb{C}} \in \mathcal{B}^{\mathrm{r}}\left(\mathcal{V}_{\mathbb{C}}\right)$.

\section{Spectrum of a Paravector}

In the complex algebra $\mathfrak{K}_{n}$, we have a natural concept of spectrum, which can be easily described in the case of paravectors. In fact, this spectrum is similar to that one introduced in [18] for quaternions. For this reason, most of the arguments used in [18] apply, with minor modificatrions, to the actual situation.

Remark 3. We follow the lines of Remark 1 from [18].

(1) Because each paravector commutes in $\mathfrak{K}_{n}$ with every complex number, we have the identities

$$
\left(\lambda-\kappa^{\star}\right)(\lambda-\kappa)=(\lambda-\kappa)\left(\lambda-\kappa^{\star}\right)=\lambda^{2}-\lambda\left(\kappa+\kappa^{\star}\right)+|\kappa|^{2} \in \mathbb{C},
$$

for all $\lambda \in \mathbb{C}$ and $\kappa \in \mathfrak{P}_{n}$. Therefore, if the complex number $\lambda^{2}-2 \lambda \Re(\kappa)+|\kappa|^{2}$ is nonnull, the element $\lambda-\kappa \in \mathfrak{K}_{n}$ is invertible. Conversely, if the element $\lambda-\kappa \in \mathfrak{K}_{n}$ is invertible, assuming $\lambda^{2}-2 \lambda \Re(\kappa)+|\kappa|^{2}=0$, the identity (3) implies $\kappa^{\star}=\lambda$. In other words, $\lambda=\kappa \in \mathbb{R}$, which is impossible because $\lambda-\kappa$ is invertible. Therefore, $\lambda-\kappa$ is invertible if and only if the complex number $\lambda^{2}-2 \lambda \Re(\kappa)+|\kappa|^{2}$ is nonnull, and thus

$$
(\lambda-\kappa)^{-1}=\frac{1}{\lambda^{2}-2 \lambda \Re(\kappa)+|\kappa|^{2}}\left(\lambda-\kappa^{\star}\right) .
$$

Hence, the element $\lambda-\kappa \in \mathfrak{K}_{n}$ is not invertible if and only if $\lambda=\Re(\kappa) \pm i|\Im(\kappa)|$. In this way, the spectrum of a paravector $\kappa$ is given by the equality $\sigma(\kappa)=\left\{s_{ \pm}(\kappa)\right\}$, where $s_{ \pm}(\kappa)=\Re(\kappa) \pm i|\Im(\kappa)|$ are the eigenvalues of $\kappa$.

The argument from above shows, in fact, that if $\lambda-\kappa$ has a left inverse, then $\lambda-\kappa$ is invertible.

(2) As usually, the resolvent set $\rho(\kappa)$ of a paravector $\kappa \in \mathfrak{P}_{n}$ is the set $\mathbb{C} \backslash \sigma(\kappa)$, while the function

$$
\rho(\kappa) \ni \lambda \mapsto(\lambda-\kappa)^{-1} \in \mathfrak{K}_{n}
$$


is the resolvent (function) of $\kappa$, which is a $\mathfrak{K}_{n}$-valued analytic function on $\rho(\kappa)$.

(3) Note that two paravectors $\kappa, \tau \in \mathfrak{P}_{n}$ have the same spectrum if and only if $\Re(\kappa)=\Re(\tau)$ and $|\Im(\kappa)|=$ $|\Im(\tau)|$.

(4) We recall that $\mathbb{S}_{n}$ is the unit sphere of purely imaginary paravectors. As already noticed, every paravector $\kappa \in \mathfrak{P}_{n} \backslash \mathbb{R}$ can be written as $\kappa=x+y \mathfrak{s}$, where $x, y$ are real numbers, with $x=\Re(\kappa), y \in\{ \pm|\Im(\kappa)|\}$, and $\mathfrak{s} \in\{ \pm \Im(\kappa) /|\Im(\kappa)|\} \subset \mathbb{S}_{n}$. Anyway, we always have $\sigma(\kappa)=\{x \pm i y\}$, because $\Im(\kappa)=y \mathfrak{s}$. Note that, for fixed real numbers $x, y$, the spectrum of $\kappa$ does not depend on $\mathfrak{s}$. Thus, for every $\lambda=u+i v \in \mathbb{C}$ with $u, v \in \mathbb{R}$, we have $\sigma(u+v \mathfrak{s})=\{\lambda, \bar{\lambda}\}$ for all $\mathfrak{s} \in \mathbb{S}_{n}$.

(5) The equality $\sigma(\kappa)=\sigma(\tau)$ is clearly an equivalence relation in $\mathfrak{P}_{n}$. It is easily seen that the equivalence class of an element $\kappa_{0}=x_{0}+y_{0} \mathfrak{s}_{0} \in \mathfrak{P}_{n}$ is given by $\left\{x_{0}+y_{0} \mathfrak{s} ; \mathfrak{s} \in \mathbb{S}_{n}\right\}$.

(6) Fixing an element $\mathfrak{s} \in \mathbb{S}_{n}$, we have an isometric $\mathbb{R}$-linear map from the complex plane $\mathbb{C}$ into the space $\mathfrak{P}_{n}$, say $\theta_{\mathfrak{s}}$, defined by $\theta_{\mathfrak{s}}(u+i v)=u+v \mathfrak{s}, u, v \in \mathbb{R}$. For every subset $A \subset \mathbb{C}$, we put

$$
A_{\mathfrak{s}}=\{x+y \mathfrak{s} ; x, y \in \mathbb{R}, x+i y \in A\}=\theta_{\mathfrak{s}}(A) .
$$

Note that, if $A$ is open in $\mathbb{C}$, then $A_{\mathfrak{s}}$ is open in the $\mathbb{R}$-vector space $\mathbb{C}_{\mathfrak{s}} \subset \mathfrak{P}_{n}$.

The corresponding version of Definition 1 from [18], adapted to the Cliffordian context, is the following.

Definition 1. The $\mathfrak{K}_{n}$-valued Cauchy kernel on the open set $\Omega \subset \mathfrak{P}_{n}$ is given by

$$
\rho(\kappa) \times \Omega \ni(\zeta, \kappa) \mapsto(\zeta-\kappa)^{-1} \in \mathfrak{K}_{n} .
$$

Recapturing Example 2 from [18], we get the following.

Example 2. The $\mathfrak{K}_{n}$-valued Cauchy kernel on the open set $\Omega \subset \mathfrak{P}_{n}$ is slice regular. Specifically, choosing an arbitrary relatively open set $V \subset \Omega \cap(\mathbb{R}+\mathbb{R} \mathfrak{s})$, and fixing $\zeta \in \cap_{\kappa \in V} \rho(\kappa)$, we can write for $\kappa=x+y \mathfrak{s} \in V$ the equalities

$$
\begin{aligned}
\frac{\partial}{\partial x}(\zeta-x-y \mathfrak{s})^{-1} & =(\zeta-x-y \mathfrak{s})^{-2}, \\
R_{\mathfrak{s}} \frac{\partial}{\partial y}(\zeta-x-y \mathfrak{s})^{-1} & =-(\zeta-x-y \mathfrak{s})^{-2},
\end{aligned}
$$

because $\mathfrak{s}^{2}=-1$, and $\zeta, \mathfrak{s}$ and $(\zeta-x-y \mathfrak{s})^{-1}$ commute in $\mathfrak{K}_{n}$. Therefore,

$$
\bar{\partial}_{\mathfrak{s}}\left((\zeta-\kappa)^{-1}\right)=\bar{\partial}_{\mathfrak{s}}\left((\zeta-x-y \mathfrak{s})^{-1}\right)=0,
$$

implying the assertion.

The next comment is inspired by Remark 2 from [18].

Remark 4. (1) The discussion about the spectrum of a paravector can be enlarged, keeping the same framework. Specifically, we may regard an element $\kappa \in \mathfrak{P}_{n}$ as a left multiplication operator on the algebra $\mathfrak{K}_{n}$, denoted by $L_{\kappa}$, defined by $L_{\kappa} \mathbf{a}=\kappa \mathbf{a}$ for all $\mathbf{a} \in \mathfrak{K}_{n}$. It is quite clear that $\sigma\left(L_{\kappa}\right)=\sigma(\kappa)$. In this context, we may find the eigenvectors of $L_{\kappa}$, which will be of interest in what follows. Therefore, we should look for solutions $v$ of the equation $\kappa v=s v$ in the algebra $\mathfrak{K}_{n}$, with $s \in \sigma(\kappa)$. Writing $\kappa=\kappa_{0}+\Im(\kappa)$ with $\kappa_{0} \in \mathbb{R}, s_{ \pm}=\kappa_{0} \pm i|\Im(\kappa)|$ and $v=\mathbf{x}+i \mathbf{y}$ with $\mathbf{x}, \mathbf{y} \in \mathfrak{C}_{n}$, we obtain the equivalent equations

$$
\Im(\kappa) \mathbf{x}=\mp|\Im(\kappa)| \mathbf{y}, \Im(\kappa) \mathbf{y}= \pm|\Im(\kappa)| \mathbf{x},
$$

leading to the solutions

$$
\nu_{ \pm}(\kappa)=\left(1 \mp i \frac{\Im(\kappa)}{|\Im(\kappa)|}\right) \mathbf{x}
$$

of the equation $\kappa v_{ \pm}=s_{ \pm} v_{ \pm}$, where $\mathbf{x} \in \mathfrak{C}_{n}$ is arbitrary, provided $\Im(\kappa) \neq 0$.

When $\Im(\kappa)=0$, the solutions are given by $v=\kappa_{0} \mathbf{a}$, with $\mathbf{a} \in \mathfrak{K}_{n}$ arbitrary. 
(2) Every paravector $\mathfrak{s} \in \mathbb{S}_{n}$ may be associated with two elements $\iota_{ \pm}(\mathfrak{s})=(1 \mp i \mathfrak{s}) / 2$ in $\mathfrak{K}_{n}$, which are commuting idempotents such that $\iota_{+}(\mathfrak{s})+\iota_{-}(\mathfrak{s})=1$ and $\iota_{+}(\mathfrak{s}) \iota_{-}(\mathfrak{s})=0$. For this reason, setting $\mathfrak{K}_{ \pm}^{\mathfrak{s}}=\iota_{ \pm}(\mathfrak{s}) \mathfrak{C}_{n}$, we have a direct sum decomposition $\mathfrak{K}_{n}=\mathfrak{K}_{+}^{\mathfrak{s}}+\mathfrak{K}_{-}^{\mathfrak{s}}$. Explicitly, if $\mathbf{a}=\mathbf{u}+i \mathbf{v}$, with $\mathbf{u}, \mathbf{v} \in \mathfrak{C}_{\mathbf{n}}$, the equation $\iota_{+}(\mathfrak{s}) \mathbf{x}+\iota_{-}(\mathfrak{s}) \mathbf{y}=\mathbf{a}$ has the unique solution $\mathbf{x}=\mathbf{u}+\mathfrak{s v}, \mathbf{y}=\mathbf{u}-\mathfrak{s v} \in \mathfrak{C}_{n}$, because $\mathfrak{s}^{-1}=-\mathfrak{s}$.

In particular, if $\kappa \in \mathfrak{P}_{n}$ and $\Im(\kappa) \neq 0$, setting $\mathfrak{s}_{\tilde{\kappa}}=\tilde{\kappa}|\tilde{\kappa}|^{-1}$, where $\tilde{\kappa}=\Im(\kappa)$, the elements $\iota_{ \pm}\left(\mathfrak{s}_{\tilde{\kappa}}\right)$ are idempotents, as above.

The next result provides explicit formulas of the spectral projections (see [4], Section VII.1) associated to the operator $L_{\kappa}, \kappa \in \mathfrak{P}_{n}$. Of course, they are not trivial only if $\kappa \in \mathfrak{P}_{n} \backslash \mathbb{R}$ because if $\kappa \in \mathbb{R}$, its spectrum is this real singleton, and the only spectral projection is the identity.

The statement of the result corresponding to Lemma 1 from [18] sounds like that:

Lemma 1. Let $\kappa \in \mathfrak{P}_{n} \backslash \mathbb{R}$ be fixed. The spectral projections associated to the eigenvalues $s_{ \pm}(\kappa)$ are given by

$$
P_{ \pm}(\kappa) \mathbf{a}=\iota_{ \pm}\left(\mathfrak{s}_{\tilde{\kappa}}\right) \mathbf{a}, \mathbf{a} \in \mathfrak{K}_{n} .
$$

Moreover, $P_{+}(\kappa) P_{-}(\kappa)=P_{-}(\kappa) P_{+}(\kappa)=0$, and $P_{+}(\kappa)+P_{-}(\kappa)$ is the identity on $\mathfrak{K}_{n}$.

When $\kappa \in \mathbb{R}$, the corresponding spectral projection is the identity on $\mathfrak{K}_{n}$.

Proof. We shall give a direct argument. Let us fix a paravector $\kappa$ with $\Im(\kappa) \neq 0$. Next, we write the general formulas for its spectral projections. Setting $s_{ \pm}=s_{ \pm}(\kappa)$, the points $s_{+}, s_{-}$are distinct and not real. We fix an $r>0$ sufficiently small such that, setting $D_{ \pm}:=\left\{\zeta \in \rho(\kappa) ;\left|\zeta-s_{ \pm}\right| \leq r\right\}$, we have $D_{ \pm} \backslash\left\{s_{ \pm}\right\} \subset \rho(\kappa)$ and $D_{+} \cap D_{-}=\emptyset$. Then the spectral projections are given by

$$
P_{ \pm}(\kappa)=\frac{1}{2 \pi i} \int_{\Gamma_{ \pm}}\left(\zeta-L_{\kappa}\right)^{-1} d \zeta
$$

where $\Gamma_{ \pm}$is the boundary of $D_{ \pm}$.

Using the equality $L_{\kappa} \nu_{ \pm}(\kappa)=s_{ \pm}(\kappa) \nu_{ \pm}(\kappa)$ (see Remark 4), for every $\zeta \in \rho(\kappa)$ and $\mathbf{h} \in \mathfrak{C}_{n}$, we obtain

$$
\left(\zeta-L_{\kappa}\right)^{-1}\left(1 \mp i \mathfrak{s}_{\tilde{\kappa}}\right) \mathbf{x}=\left(\zeta-S_{ \pm}\right)^{-1}\left(1 \mp i \mathfrak{s}_{\tilde{\kappa}}\right) \mathbf{x},
$$

by Remark 4. Therefore,

$$
P_{+}(\kappa)\left(1 \mp i \mathfrak{s}_{\tilde{\kappa}}\right) \mathbf{x}=\frac{1}{2 \pi i} \int_{\Gamma_{+}}\left(\zeta-s_{ \pm}\right)^{-1}\left(1 \mp i \mathfrak{s}_{\tilde{\kappa}}\right) \mathbf{x} d \zeta
$$

and

$$
P_{-}(\kappa)\left(1 \mp i \mathfrak{s}_{\tilde{\kappa}}\right) \mathbf{x}=\frac{1}{2 \pi i} \int_{\Gamma_{-}}\left(\zeta-s_{ \pm}\right)^{-1}\left(1 \mp i \mathfrak{s}_{\tilde{\kappa}}\right) \mathbf{x} d \zeta .
$$

Using Cauchy’s formula, we deduce that

$$
P_{+}(\kappa)\left(1-i \mathfrak{s}_{\tilde{\kappa}}\right) \mathbf{x}=\left(1-i \mathfrak{s}_{\tilde{\kappa}}\right) \mathbf{x}, P_{+}(\kappa)\left(1+i \mathfrak{s}_{\tilde{\kappa}}\right) \mathbf{x}=0,
$$

and

$$
P_{-}(\kappa)\left(1-i \mathfrak{s}_{\tilde{\kappa}}\right) \mathbf{x}=0, P_{-}(\kappa)\left(1+i \mathfrak{s}_{\tilde{\kappa}}\right) \mathbf{x}=\left(1+i \mathfrak{s}_{\tilde{\kappa}}\right) \mathbf{x},
$$

for all $\mathbf{x} \in \mathfrak{C}_{n}$.

Fixing an arbitrary element $\mathbf{a}=\mathbf{u}+i \mathbf{v} \in \mathfrak{K}_{n}$ and noticing that $P_{ \pm}(\kappa)$ are $\mathbb{C}$-linear, we clearly obtain $P_{ \pm}(\kappa) \mathbf{a}=\iota_{ \pm}\left(\mathfrak{s}_{\tilde{\kappa}}\right) \mathbf{a}, \mathbf{a} \in \mathfrak{K}_{n}$, which are precisely the formulas (6) from the statement.

The properties $P_{+}(\kappa) P_{-}(\kappa)=P_{-}(\kappa) P_{+}(\kappa)=0$, and $P_{+}(\kappa)+P_{-}(\kappa)$ is the identity on $\mathfrak{K}_{n}$ are direct consequences of the analytic functional calculus associated to a fixed element $\kappa \in \mathfrak{P}_{n}$ in the algebra $\mathfrak{K}_{n}$.

By a slight abuse of terminology, the projections $P_{ \pm}(\kappa)$ will be also called the spectral projections of $\kappa$. In fact, as formula (6) shows, they depend only on the imaginary part $\tilde{\kappa}$ of $\kappa$. 
Corollary 1. For every $\kappa \in \mathfrak{P}_{n}$ and $\mathbf{a} \in \mathfrak{K}_{n}$ we have

$$
L_{\kappa} \mathbf{a}=s_{+}(\kappa) P_{+}(\kappa) \mathbf{a}+s_{-}(\kappa) P_{-}(\kappa) \mathbf{a} .
$$

Proof Assume first that $\kappa \in \mathfrak{P}_{n} \backslash \mathbb{R}$ and $\mathbf{a} \in \mathfrak{C}_{n}$. Then formula (6) implies that

$$
\mathbf{a}=P_{+}(\kappa) \mathbf{a}+P_{-}(\kappa) \mathbf{a}=\iota_{+}\left(\mathfrak{s}_{\tilde{\kappa}}\right) \mathbf{a}+\iota_{-}\left(\mathfrak{s}_{\tilde{\kappa}}\right) \mathbf{a} .
$$

Therefore,

$$
\begin{gathered}
L_{\kappa} \mathbf{a}=\kappa \iota_{+}\left(\mathfrak{s}_{\tilde{\kappa}}\right) \mathbf{a}+\kappa \iota_{-}\left(\mathfrak{s}_{\tilde{\kappa}}\right) \mathbf{a}=s_{+}(\kappa) \iota_{+}\left(\mathfrak{s}_{\tilde{\kappa}}\right) \mathbf{a}+s_{-}(\kappa) \iota_{-}\left(\mathfrak{s}_{\tilde{\kappa}}\right) \mathbf{a}= \\
s_{+}(\kappa) P_{+}(\kappa) \mathbf{a}+s_{-}(\kappa) P_{-}(\kappa) \mathbf{a} .
\end{gathered}
$$

This formula can be extended to elements of $\mathfrak{K}_{n}$ because all operations within are $\mathbb{C}$-linear.

Of course, this formula also holds for $\kappa=r \in \mathbb{R}$, leading to $L_{r} \mathbf{a}=r \mathbf{a}$

\section{A General Functional Calculus for Stem Functions}

In this section, some spaces of $\mathfrak{K}_{n}$-valued functions, defined on subsets of the complex plane, will be associated with spaces of functions, defined on subsets of $\mathfrak{P}_{n}$, taking values in the Clifford algebra $\mathfrak{C}_{n}$, using spectral methods and functional calculi. As in [18] (in the context of quaternionic valued functions), this is a general functional calculus, with arbitrary functions (see Theorem 2).

Remark 5. Following [18], Remark 3, an idea from the theory of spectral operators, developed in [4], Part III, can be applied to define an appropriate functional calculus, also useful in the Cliffordian context. Specifically, regarding the algebra $\mathfrak{K}_{n}$ as a (complex) Banach space, and denoting by $\mathcal{B}\left(\mathfrak{K}_{n}\right)$ the Banach space of all linear operators acting on $\mathfrak{K}_{n}$, the operator $L_{\kappa}, \kappa \in \mathfrak{P}_{n}$, (see Remark 4(1)) is a particular case of a scalar type operator, as defined in [4], Part III, XV.4.1. Its resolution of the identity consists of four projections $\left\{0, P_{ \pm}(\kappa), \mathrm{I}\right\}$, including the null operator 0 and the identity I, where $P_{ \pm}(\kappa)$ are the spectral projections of $L_{\kappa}$, and its integral representation is given by

$$
L_{\kappa}=s_{+}(\kappa) P_{+}(\kappa)+s_{-}(\kappa) P_{-}(\kappa) \in \mathcal{B}\left(\mathfrak{K}_{n}\right),
$$

provided by Corollary 1 .

For every function $f: \sigma(\kappa) \mapsto \mathbb{C}$ we may define the operator

$$
f\left(L_{\kappa}\right)=f\left(s_{+}(\kappa)\right) P_{+}(\kappa)+f\left(s_{-}(\kappa)\right) P_{-}(\kappa) \in \mathcal{B}\left(\mathfrak{K}_{n}\right) .
$$

which provides a functional calculus with arbitrary functions on the spectrum. More generally, regarding the elements of the algebra $\mathfrak{K}_{n}$ as left multiplication operators on $\mathfrak{K}_{n}$, we may extend this formula to functions of the form $F: \sigma(\kappa) \mapsto \mathcal{B}\left(\mathfrak{K}_{n}\right)$, putting

$$
F\left(L_{\kappa}\right)=F\left(s_{+}(\kappa)\right) P_{+}(\kappa)+F\left(s_{-}(\kappa)\right) P_{-}(\kappa),
$$

and keeping this order, which is a "left functional calculus“, not multiplicative, in general. It is this idea which leads us to try to define some $\mathfrak{C}_{n}$-valued functions on subsets of $\mathfrak{P}_{n}$ associated to certain $\mathfrak{K}_{n}$-valued functions, defined on some subsets of $\mathbb{C}$.

Definition 2. (1) A subset $S \subset \mathbb{C}$ is said to be conjugate symmetric if $\zeta \in S$ if and only if $\bar{\zeta} \in S$.

(2) A subset $A \subset \mathfrak{P}_{n}$ is said to be spectrally saturated (as in [18]) if whenever $\sigma(\theta)=\sigma(\kappa)$ for some $\theta \in \mathfrak{P}_{n}$ and $\kappa \in A$, we also have $\theta \in A$.

For an arbitrary $A \subset \mathfrak{P}_{n}$, we put $\mathfrak{S}(A)=\cup_{\kappa \in A} \sigma(\kappa) \subset \mathbb{C}$.

Conversely, for an arbitrary subset $S \subset \mathbb{C}$, we put $S_{\sigma}=\left\{\kappa \in \mathfrak{P}_{n} ; \sigma(\kappa) \subset S\right\}$.

The discussion from [18], Remark 4, can be adapted to this context in the following way. 
Remark 6. (1) If $A \subset \mathfrak{P}_{n}$ is spectrally saturated, then $S=\mathfrak{S}(A)$ is conjugate symmetric, and conversely, if $S \subset \mathbb{C}$ is conjugate symmetric, then $S_{\sigma}$ is spectrally saturated, which can be easily seen. Moreover, the assignment $S \mapsto S_{\sigma}$ is injective. Indeed, if $\lambda=u+i v \in S, u, v \in \mathbb{R}$, then $\lambda \in \sigma(u+v \mathfrak{s})$ for a fixed $\mathfrak{s} \in \mathbb{S}_{n}$. If $S_{\sigma}=T_{\sigma}$ for some $T \subset \mathbb{C}$, we must have $\kappa=u+v \mathfrak{s} \in T_{\sigma}$. Therefore $\sigma(u+v \mathfrak{s}) \subset T$, implying $\lambda \in T$, and so $S \subset T$. Clearly, we also have $T \subset S$.

Similarly, the assignment $A \mapsto \mathfrak{S}(A)$ is injective and $A=S_{\sigma}$ if and only if $S=\mathfrak{S}(A)$. These two assertions are left to the reader.

(2) If $\Omega \subset \mathfrak{P}_{n}$ is an open spectraly saturated set, then $\mathfrak{S}(\Omega) \subset \mathbb{C}$ is open. To see that, let $\lambda_{0}=u_{0}+i v_{0} \in$ $\mathfrak{S}(\Omega)$ be fixed, with $u_{0}, v_{0} \in \mathbb{R}$, and let $\kappa_{0}=u_{0}+v_{0} \mathfrak{s}$, where $\mathfrak{s} \in \mathbb{S}_{n}$ is also fixed. Because $\Omega$ is spectrally saturated, we must have $\kappa_{0} \in \Omega$. As the set $\Omega \cap \mathbb{C}_{\mathfrak{s}}$ is relatively open, there is a positive number $r$ such that the open set

$$
\left\{\kappa=u+v \mathfrak{s} ; u, v \in \mathbb{R},\left|\kappa-\kappa_{0}\right|<r\right\}
$$

is in $\Omega \cap \mathbb{C}_{\mathfrak{s}}$. Therefore, the set of the points $\lambda=u+i v$, satisfying $\left|\lambda-\lambda_{0}\right|<r$ is in $\mathfrak{S}(\Omega)$, implying that it is open.

Conversely, if $U \subset \mathbb{C}$ is open and conjugate symmetric, the set $U_{\sigma}$ is also open via the upper semicontinuity of the spectrum (see [4], Part I, Lemma VII.6.3.).

An important particular case is when $U=\mathbb{D}_{r}:=\{\zeta \in \mathbb{C} ;|\zeta|<r\}$, for some $r>0$. Then $U_{\sigma}=\left\{\kappa \in \mathfrak{P}_{n} ;|\kappa|<\right.$ $r\}$. Indeed, if $|\kappa|<r$ and $\theta$ has the property $\sigma(\kappa)=\sigma(\theta)$, from the equality $\{\Re(\kappa) \pm i|\Im(\kappa)|\}=\{\Re(\theta) \pm i|\Im(\theta)|\}$ it follows that $|\theta|<r$.

(3) A subset $\Omega \subset \mathfrak{C}_{n}$ is said to be axially symmetric if for every $\kappa_{0}=u_{0}+v_{0} \mathfrak{s}_{0} \in \Omega$ with $u_{0}, v_{0} \in \mathbb{R}$ and $\mathfrak{s}_{0} \in \mathbb{S}_{n}$, we also have $\kappa=u_{0}+v_{0} \mathfrak{s} \in \Omega$ for all $\mathfrak{s} \in \mathbb{S}_{n}$. This concept is introduced in [2], Definition 2.2.17. In fact, we have the following.

Lemma 2. A subset $\Omega \subset \mathfrak{C}_{n}$ is axially symmetric if and only if it is spectrally saturated.

The assertion follows easily from the fact that the equality $\sigma(\kappa)=\sigma(\tau)$ is an equivalence relation in $\mathfrak{P}_{n}$ (see Remark 3(5)).

Nevertheless, we continue to use the expression "spectrally saturated set“ to designate an "axially symmetric set“, because the former name is more compatible with our spectral approach.

As noticed in Remark 1, the algebra $\mathfrak{K}_{n}$ is endowed with a conjugation given by $\overline{\mathbf{a}}=\mathbf{b}-i \mathbf{c}$, when $\mathbf{a}=\mathbf{b}+i \mathbf{c}$, with b, $\mathbf{c} \in \mathfrak{C}_{n}$. Note also that, because $\mathbb{C}$ is a subalgebra of $\mathfrak{K}_{n}$, the conjugation of $\mathfrak{K}_{n}$ restricted to $\mathbb{C}$ is precisely the usual complex conjugation.

The next definition has an old origin, seemingly going back to [6].

Definition 3. Let $U \subset \mathbb{C}$ be conjugate symmetric, and let $F: U \mapsto \mathfrak{K}_{n}$. We say that $F$ is a $\left(\mathfrak{K}_{n}\right.$-valued) stem function if $F(\bar{\lambda})=\overline{F(\lambda)}$ for all $\lambda \in U$.

For an arbitrary conjugate symmetric subset $U \subset \mathbb{C}$, we put

$$
\mathcal{S}\left(U, \mathfrak{K}_{n}\right)=\left\{F: U \mapsto \mathfrak{K}_{n} ; F(\bar{\zeta})=\overline{F(\zeta)}, \zeta \in U\right\},
$$

that is, the $\mathbb{R}$-vector space of all $\mathfrak{K}_{n}$-valued stem functions on $U$. Replacing $\mathfrak{K}_{n}$ by $\mathbb{C}$, we denote by $\mathcal{S}(U)$ the real algebra of all $\mathbb{C}$-valued stem functions, which is an $\mathbb{R}$-subalgebra in $\mathcal{S}\left(U, \mathfrak{K}_{n}\right)$. In addition, the space $\mathcal{S}\left(U, \mathfrak{K}_{n}\right)$ is a two-sided $\mathcal{S}(U)$-module.

The following definition adapts, to our context, Definition 4 from [18].

Definition 4. Let $U \subset \mathbb{C}$ be conjugate symmetric. For every $F: U \mapsto \mathfrak{K}_{n}$ we define a function $F_{\sigma}: U_{\sigma} \mapsto \mathfrak{K}_{n}$, via the assignment

$$
U_{\sigma} \backslash \mathbb{R} \ni \kappa \mapsto F_{\sigma}(\kappa)=F\left(s_{+}(\kappa)\right) \iota_{+}\left(\mathfrak{s}_{\tilde{\kappa}}\right)+F\left(s_{-}(\kappa)\right) \iota_{-}\left(\mathfrak{s}_{\tilde{\kappa}}\right) \in \mathfrak{K}_{n},
$$

where $\tilde{\kappa}=\Im(\kappa), \mathfrak{s}_{\tilde{\kappa}}=|\tilde{\kappa}|^{-1} \tilde{\kappa}$, and $\iota_{ \pm}\left(\mathfrak{s}_{\tilde{\kappa}}\right)=2^{-1}\left(1 \mp i \mathfrak{s}_{\tilde{\kappa}}\right)$, and $F_{\sigma}(r)=F(r)$, if $r \in U_{\sigma} \cap \mathbb{R}$. 
Formula (9) is strongly related to formula (7) because the spectral projections $P_{ \pm}(\kappa)$ are the left multiplications defined by $2^{-1}\left(1 \mp i \mathfrak{s}_{\tilde{\kappa}}\right)$ respectively, via formula (6).

The next result is a version of Theorem 2 from [18].

Theorem 1. Let $U \subset \mathbb{C}$ be a conjugate symmetric subset, and let $F: U \mapsto \mathfrak{K}_{n}$. The element $F_{\sigma}(\kappa)$ belongs to $\mathfrak{C}_{n}$ for all $\kappa \in U_{\sigma}$ if and only if $F \in \mathcal{S}\left(U, \mathfrak{K}_{n}\right)$.

Proof. We first assume that $F_{\sigma}(\kappa) \in \mathfrak{C}_{n}$ for all $\kappa \in U_{\sigma}$. We fix a point $\zeta \in U$, supposing that $\Im \zeta>0$. Then we choose a paravector $\kappa \in U_{\sigma}$ with $\sigma(\kappa)=\{\zeta, \bar{\zeta}\}$. Therefore, $s_{+}(\kappa)=\zeta$ and $s_{-}(\kappa)=\bar{\zeta}$. We write $F(\zeta)=$ $F_{+1}+i F_{+2}, F(\bar{\zeta})=F_{-1}+i F_{-2}$, with $F_{+1}, F_{+2}, F_{-1}, F_{-2} \in \mathfrak{C}_{n}$. According to (9), we have

$$
2 F(\kappa)=F_{+1}+F_{+2} \mathfrak{s}_{\tilde{\kappa}}+F_{-1}-F_{-2} \mathfrak{s}_{\tilde{\mathcal{K}}}+i\left(-F_{+1} \mathfrak{s}_{\tilde{\mathcal{K}}}+F_{+2}+F_{-2}+F_{-1} \mathfrak{s}_{\tilde{\mathcal{K}}}\right),
$$

so

$$
2 \overline{F(\kappa)}=F_{+1}+F_{+2} \mathfrak{s}_{\tilde{\kappa}}+F_{-1}-F_{-2} \mathfrak{s}_{\tilde{\kappa}}+i\left(F_{+1} \mathfrak{s}_{\tilde{\kappa}}-F_{+2}-F_{-2}-F_{-1} \mathfrak{s}_{\tilde{\kappa}}\right) .
$$

Because $F(\kappa)=\overline{F(\kappa)}$, we must have

$$
-F_{+1} \mathfrak{s}_{\tilde{\mathcal{K}}}+F_{+2}+F_{-2}+F_{-1} \mathfrak{s}_{\tilde{\mathcal{K}}}=F_{+1} \mathfrak{S}_{\tilde{\mathcal{K}}}-F_{+2}-F_{-2}-F_{-1} \mathfrak{s}_{\tilde{\kappa}},
$$

which is equivalent to

$$
F_{+2}+F_{-2}=\left(F_{+1}-F_{-1}\right) \mathfrak{s}_{\tilde{\kappa}}
$$

Choosing another paravector $\theta \in \mathfrak{P}_{n}$ with $s_{+}(\theta)=\zeta$ but with $\mathfrak{s}_{\tilde{\kappa}} \neq \mathfrak{s}_{\tilde{\theta}}$, we obtain

$$
\left(F_{+1}-F_{-1}\right)\left(\mathfrak{s}_{\tilde{\kappa}}-\mathfrak{s}_{\tilde{\theta}}\right)=0 .
$$

Because the paravector $\mathfrak{s}_{\tilde{\kappa}}-\mathfrak{s}_{\tilde{\theta}}$ is nonnull, it must be invertible, and so $F_{+1}=F_{-1}$, implying $F_{+2}=-F_{-2}$, meaning that $\overline{F(\zeta)}=F(\bar{\zeta})$.

If $\Im \zeta=0$, so $\zeta=x_{0} \in \mathbb{R}$, taking $\kappa=x_{0}$, we have $\sigma(\kappa)=\left\{x_{0}\right\}$, and $F(\kappa)=F\left(x_{0}\right)$ is a real number. $U$.

If $\Im \zeta<0$, applying the above argument to $\bar{\zeta}$ we obtain $\overline{F(\bar{\zeta})}=F(\zeta)$. Consequently, $F$ is a stem function on

Conversely, if $\overline{F(\zeta)}=F(\bar{\zeta})$ for all $\zeta \in U$, choosing a $\kappa \in \mathfrak{P}_{n}$ with $\Im \kappa \neq 0$, and setting $\zeta=s_{+}(\kappa)$, we obtain from (9) the equality

$$
2 F_{\sigma}(\kappa)=F(\zeta)+F(\bar{\zeta})-i\left(F(\zeta)-F(\bar{\zeta}) \mathfrak{s}_{\tilde{\kappa}} .\right.
$$

Therefore,

$$
\left.2 \overline{F_{\sigma}(\kappa)}=F(\bar{\zeta})+F(\zeta)+i(F(\bar{\zeta})-F(\zeta))\right)_{\tilde{\kappa}},
$$

showing that $F_{\sigma}(\kappa) \in \mathfrak{C}_{n}$ for all $\kappa \in U_{\sigma}$, because the case $\kappa=r \in \mathbb{R}$ is obvious.

Corollary 2. Let $U \subset \mathbb{C}$ be a conjugate symmetric subset, and let $f: U \mapsto \mathbb{C}$. The following conditions are equivalent;

(1) $f \in \mathcal{S}(U)$;

(2) $f_{\sigma}(\kappa)$ belongs to $\mathbb{C}_{\mathfrak{s}}$, and $f_{\sigma}\left(\kappa^{\star}\right)=f_{\sigma}(\kappa)^{\star}$ for all $\kappa \in U_{\sigma} \cap \mathbb{C}_{\mathfrak{s}}$, where $\mathbb{C}_{\mathfrak{s}}=\{u+v \mathfrak{s} ; u, v \in \mathbb{R}\}$, and $\mathfrak{s} \in \mathbb{S}_{n}$.

Proof. That $f \in \mathcal{S}(U)$ implies $f_{\sigma}(\kappa)$ belongs to $\mathfrak{C}_{n}$ for all $\kappa \in U_{\sigma}$ is a direct consequence of Theorem 1. More precisely, in this case actually (2) holds true. To get (2), let us first choose a function $f \in \mathcal{S}(U)$. It follows from Definition 4 that

$$
\begin{gathered}
f_{\sigma}(\kappa)=f\left(s_{+}(\kappa)\right) \iota_{+}\left(\mathfrak{s}_{\tilde{\kappa}}\right)+\overline{f\left(s_{+}(\kappa)\right)} \iota_{-}\left(\mathfrak{s}_{\tilde{\kappa}}\right)= \\
\Re\left(f\left(s_{+}(\kappa)\right)+\Im\left(f\left(s_{+}(\kappa)\right) \mathfrak{s}_{\tilde{\kappa}} \in \mathbb{C}_{\mathfrak{s}_{\tilde{\kappa}}}\right.\right.
\end{gathered}
$$

where $\kappa \in U_{\sigma}, \tilde{\kappa}=\Im(\kappa), \mathfrak{s}_{\tilde{\kappa}}=\tilde{\kappa}|\tilde{\kappa}|^{-1}$, and $\iota_{ \pm}\left(\mathfrak{s}_{\tilde{\kappa}}\right)=2^{-1}\left(1 \mp i \mathfrak{s}_{\tilde{\kappa}}\right)$. Because $s_{+}\left(\kappa^{\star}\right)=s_{+}(\kappa)$, and $\tilde{\kappa}^{\star}=-\tilde{\kappa}$, we clearly have $f_{\sigma}\left(\kappa^{\star}\right)=f_{\sigma}(\kappa)^{\star}$ for all $\kappa \in U_{\sigma}$. 
Conversely, let $g: U_{\sigma} \mapsto \mathfrak{P}_{n}$ be such that $g(\kappa) \in \mathbb{C}_{\mathfrak{s}}$, and $g\left(\kappa^{\star}\right)=g(\kappa)^{\star}$ for all $\kappa \in U_{\sigma} \cap \mathbb{C}_{\mathfrak{s}}$. We shall look for a function $f \in \mathcal{S}(U)$ such that $f_{\sigma}=g$. Fixing the points $z_{ \pm}=x \pm i y \in U$ with $x, y(\neq 0) \in \mathbb{R}$, and a paravector $\mathfrak{s} \in \mathbb{S}_{n}$, we set $\kappa_{ \pm}=x \pm y \mathfrak{s}$, so $\kappa_{ \pm}^{\star}=\kappa_{\mp}, s_{+}\left(\kappa_{ \pm}\right)=x+i|y|$, and $s_{-}\left(\kappa_{ \pm}\right)=x-i|y|$. Then we define

$$
\begin{aligned}
& 2 f\left(z_{+}\right)=g\left(\kappa_{+}\right)(1-i \mathfrak{s})+g\left(\kappa_{-}\right)(1+i \mathfrak{s}), \\
& 2 f\left(z_{-}\right)=g\left(\kappa_{+}\right)(1+i \mathfrak{s})+g\left(\kappa_{-}\right)(1-i \mathfrak{s}) .
\end{aligned}
$$

Because $g\left(\kappa_{+}\right) \in \mathbb{C}_{\mathfrak{s}}$, there are $u, v \in \mathbb{R}$ such that $g\left(\kappa_{+}\right)=u+v \mathfrak{s}$, and thus, $g\left(\kappa_{-}\right)=u-v \mathfrak{s}$. Therefore, $f\left(z_{+}\right)=u+i v \in \mathbb{C}$. Similarly, $f\left(z_{-}\right)=u-i v \in \mathbb{C}$. This shows, in fact, that $f: U \mapsto \mathbb{C}$, and $f(z)=\overline{f(\bar{z})}$. Using the equations from above, we derive easily that

$$
\begin{aligned}
& 2 g\left(\kappa_{+}\right)=f\left(z_{+}\right)(1-i \mathfrak{s})+f\left(z_{-}\right)(1+i \mathfrak{s}), \\
& 2 g\left(\kappa_{-}\right)=f\left(z_{+}\right)\left((1+i \mathfrak{s})+\left(z_{-}\right)(1-i \mathfrak{s}) .\right.
\end{aligned}
$$

implying the equality $g(\kappa)=f_{\sigma}(\kappa)$ for all $\kappa \in U_{\sigma}$, which concludes the proof.

Remark 7. This is a description of all zeros of the functions obtained via Theorem 1, corresponding to Remark 5 from [18].

Let $U \subset \mathbb{C}$ be a conjugate symmetric set and let $F \in \mathcal{S}\left(U, \mathfrak{K}_{n}\right)$ be arbitrary. We can easily describe the zeros of $F_{\sigma}$. Indeed, if $F_{\sigma}(\kappa)=F\left(s_{+}(\kappa)\right) \iota_{+}(\tilde{\kappa})+F\left(s_{-}(\kappa)\right) \iota_{-}(\tilde{\kappa})=0$, we must have $F\left(s_{+}(\kappa)\right) \iota_{+}(\tilde{\kappa})=0$ and $F\left(s_{-}(\kappa)\right) \iota_{-}(\tilde{\kappa})=0$, via a direct manipulation with the idempotents $\iota_{ \pm}(\tilde{\kappa})$. In other words, we must have $\left.F\left(s_{ \pm}(\kappa)\right)= \pm i F\left(s_{ \pm}(\kappa)\right)\right)_{\tilde{s}}$. As in the previous proof, choosing another paravector $\theta$ with $s_{+}(\kappa) \in \sigma(\theta)$ and $\tilde{\kappa} \neq \tilde{\theta}$, we obtain $F\left(s_{+}(\kappa)\right)\left(\mathfrak{s}_{\tilde{\kappa}}-\right.$ $\left.\mathfrak{s}_{\tilde{\theta}}\right)=0$. Therefore, $F\left(s_{+}(\kappa)\right)=0$ because $\mathfrak{s}_{\tilde{\kappa}}-\mathfrak{s}_{\tilde{\theta}}$ is invertible. Similarly, $F\left(s_{-}(\kappa)\right)=0$. Conqequently, setting $z(F):=\{\lambda \in U ; F(\lambda)=0\}$, and $z\left(F_{\sigma}\right):=\left\{\kappa \in U_{\sigma} ; F_{\sigma}(\kappa)=0\right\}$, we must have

$$
Z\left(F_{\sigma}\right)=\left\{\kappa \in U_{\sigma} ; \sigma(\kappa) \subset Z(F)\right\} .
$$

For every subset $\Omega \subset \mathfrak{P}_{n}$, we denote by $\mathcal{F}\left(\Omega, \mathfrak{C}_{n}\right)$ the set of all $\mathfrak{C}_{n}$-valued functions on $\Omega$. Let also

$$
\mathcal{J F}\left(\Omega, \mathfrak{C}_{n}\right)=\left\{g: \mathcal{F}\left(\Omega, \mathfrak{C}_{n}\right) ; g\left(\kappa^{*}\right)=g(\kappa)^{\star} \in \mathbb{C}_{\mathfrak{s}}, \kappa \in \Omega \cap \mathbb{C}_{\mathfrak{s}}, \mathfrak{s} \in \mathbb{S}_{n}\right\},
$$

which is a unital commutative subalgebra of the algebra $\mathcal{F}\left(\Omega, \mathfrak{C}_{n}\right)$. The functions from the space $\mathcal{J F}\left(\Omega, \mathfrak{P}_{n}\right)$ are similar to those called intrinsic functions, appering in [2], Definition 3.5.1, or in [3], Definition 2.1.2.

The next result provides a $\mathfrak{C}_{n}$-valued general functional calculus for arbitrary stem functions (for the quaternionic case see [18], Theorem 2).

Theorem 2. Let $\Omega \subset \mathfrak{P}_{n}$ be a spectrally saturated set, and let $U=\mathfrak{S}(\Omega)$. The map

$$
\mathcal{S}\left(U, \mathfrak{K}_{n}\right) \ni F \mapsto F_{\sigma} \in \mathcal{F}\left(\Omega, \mathfrak{C}_{n}\right)
$$

is $\mathbb{R}$-linear, injective, and has the property $(F f)_{\sigma}=F_{\sigma} f_{\sigma}$ for all $F \in \mathcal{S}\left(U, \mathfrak{K}_{n}\right)$ and $f \in \mathcal{S}(U)$. Moreover, the restricted map

$$
\mathcal{S}(U) \ni f \mapsto f_{\sigma} \in \mathcal{J F}\left(\Omega, \mathfrak{C}_{n}\right)
$$

is unital and multiplicative.

Proof. The map $F \mapsto F_{\sigma}$ is clearly $\mathbb{R}$-linear. The injectivity of this map follows from Remark 7 . Note also that

$$
\begin{gathered}
F_{\sigma}(\kappa) f_{\sigma}(\kappa)=\left(F\left(s_{+}(\kappa)\right) \iota_{+}\left(\mathfrak{s}_{\tilde{\kappa}}\right)+F\left(s_{-}(\kappa)\right) \iota_{-}\left(\mathfrak{s}_{\tilde{\kappa}}\right)\right) \times \\
\left(f\left(s_{+}(\kappa)\right) \iota_{+}\left(\mathfrak{s}_{\tilde{\kappa}}\right)+f\left(s_{-}(\kappa)\right) \iota_{-}\left(\mathfrak{s}_{\tilde{\kappa}}\right)=\right. \\
(F f)\left(s_{+}(\kappa)\right) \iota_{+}\left(\mathfrak{s}_{\tilde{\kappa}}\right)+(F f)\left(s_{-}(\kappa)\right) \iota_{-}\left(\mathfrak{s}_{\tilde{\kappa}}\right)=(F f){ }_{\sigma}(\kappa),
\end{gathered}
$$

because $f$ is complex valued, and using the properties of the idempotents $\iota_{ \pm}\left(\mathfrak{s}_{\tilde{\kappa}}\right)$ In particular, this computation shows that if $f, g \in \mathcal{S}(U)$, and so $f_{\sigma}, g_{\sigma} \in \mathcal{J F}\left(\Omega, \mathfrak{C}_{n}\right)$ by Corollary 2, we have $(f g)_{\sigma}=f_{\sigma} g_{\sigma}=g_{\sigma} f_{\sigma}$, thus the $\operatorname{map} f \mapsto f_{\sigma}$ is multiplicative. It is also clearly unital. 


\section{A Cauchy Transform in the Clifford Algebra Context}

Having the $\mathfrak{K}_{n}$-valued Cauchy kernel (see Definition 1), we may introduce a concept of a Cauchy transform (as in [18] in the quaternionic context), whose some useful properties will be exibited in this section.

The frequent use of versions of the Cauchy formula is simplified by adopting the following definition. Let $U \subset \mathbb{C}$ be open. An open subset $\Delta \subset U$ will be called a Cauchy domain (in $U$ ) if $\Delta \subset \bar{\Delta} \subset U$ and the boundary $\partial \Delta$ of $\Delta$ consists of a finite family of closed curves, piecewise smooth, positively oriented. A Cauchy domain is bounded but not necessarily connected.

For a given open set $U \subset \mathbb{C}$, we denote by $\mathcal{O}\left(U, \mathfrak{K}_{n}\right)$ the complex algebra of all $\mathfrak{K}_{n}$-valued analytic functions on $U$.

If $U \subset \mathbb{C}$ is also conjugate symmetric, let $\mathcal{O}_{s}\left(U, \mathfrak{K}_{n}\right)$ be the real subalgebra of $\mathcal{O}\left(U, \mathfrak{K}_{n}\right)$ consisting of all stem functions from $\mathcal{O}\left(U, \mathfrak{K}_{n}\right)$.

Because $\mathbb{C} \subset \mathfrak{K}_{n}$, we have $\mathcal{O}(U) \subset \mathcal{O}\left(U, \mathfrak{K}_{n}\right)$, where $\mathcal{O}(U)$ is the complex algebra of all complex-valued analytic functions on the open set $U$. Similarly, when $U \subset \mathbb{C}$ is also conjugate symmetric, $\mathcal{O}_{s}(U) \subset \mathcal{O}_{s}\left(U, \mathfrak{K}_{n}\right)$, where $\mathcal{O}_{s}(U)$ is the real subalgebra consisting of all functions $f$ from $\mathcal{O}(U)$ which are stem functions.

As un example, if $\Delta \subset \mathbb{C}$ is an open disk centered at 0 , each function $F \in \mathcal{O}_{s}\left(\Delta, \mathfrak{K}_{n}\right)$ can be represented as a convergent series $F(\zeta)=\sum_{k \geq 0} a_{k} \zeta^{k}, \zeta \in \Delta$, with $a_{k} \in \mathfrak{C}_{n}$ for all $k \geq 0$.

Definition 5. Let $U \subset \mathbb{C}$ be a conjugate symmetric open set, and let $F \in \mathcal{O}\left(U, \mathfrak{K}_{n}\right)$. For every $\kappa \in U_{\sigma}$ we set

$$
C[F](\kappa)=\frac{1}{2 \pi i} \int_{\Gamma} F(\zeta)(\zeta-\kappa)^{-1} d \zeta,
$$

where $\Gamma$ is the boundary of a Cauchy domain in $U$ containing the spectrum $\sigma(\kappa)$. The function $C[F]: U_{\sigma} \mapsto \mathfrak{K}_{n}$ is called the ( $\mathfrak{K}_{n}$-valued) Cauchy transform of the function $F \in \mathcal{O}\left(U, \mathfrak{K}_{n}\right)$. Clearly, the function $C[F]$ does not depend on the choice of $\Gamma$ because the function $U \backslash \sigma(\kappa) \ni \zeta \mapsto F(\zeta)(\zeta-\kappa)^{-1} \in \mathfrak{K}_{n}$ is analytic.

We shall put

$$
\mathcal{R}\left(U_{\sigma}, \mathfrak{K}_{n}\right)=\left\{C[F] ; F \in \mathcal{O}\left(U, \mathfrak{K}_{n}\right)\right\} .
$$

Proposition 1. Let $U \subset \mathbb{C}$ be open and conjugate symmetric, and let $F \in \mathcal{O}\left(U, \mathfrak{K}_{n}\right)$. Then function $C[F] \in$ $\mathcal{R}\left(U_{\sigma}, \mathfrak{K}_{n}\right)$ is slice regular on $U_{\sigma}$.

Proof. Let $F \in \mathcal{O}\left(U, \mathfrak{K}_{n}\right)$, let $\kappa \in U_{\sigma}$ and let $\Delta \ni \sigma(\kappa)$ be a conjugate symmetric Cauchy domain in $U$, whose boundary is denoted by $\Gamma$. We use the representation of $C[F](\kappa)$ given by (11). Because we have

$$
\bar{\partial}_{\mathfrak{s}}\left((\zeta-\kappa)^{-1}\right)=\bar{\partial}_{\mathfrak{s}}\left((\zeta-x-y \mathfrak{s})^{-1}\right)=0
$$

for $\kappa=x+y \mathfrak{s} \in \Delta_{\sigma} \cap(\mathbb{R}+\mathbb{R} \mathfrak{s})$, via Example 2, we infer that

$$
\bar{\partial}_{\mathfrak{s}}(C[F](\kappa))=\frac{1}{2 \pi i} \int_{\Gamma} F(\zeta) \bar{\partial}_{\mathfrak{s}}\left((\zeta-\kappa)^{-1}\right) d \zeta=0,
$$

which implies the assertion.

Remark 8. (1) Because the function $F$ does not necessarily commute with the left multiplication by $\mathfrak{s} \in \mathbb{S}_{n}$, the choice of the right multiplication in the slice regularity is necessary to get the stated property of $C[F]$.

(2) Let $r>0$ and let $U \supset\{\zeta \in \mathbb{C} ;|\zeta| \leq r\}$ be a conjugate symmetric open set. Then for every $F \in \mathcal{O}\left(U, \mathfrak{K}_{n}\right)$ one has

$$
C[F](\kappa)=\sum_{n \geq 0} \frac{F^{(n)}(0)}{n !} \kappa^{n},|\kappa|<r,
$$

where the series is absolutely convergent. Of course, using the convergent series $(\zeta-\kappa)^{-1}=\sum_{n \geq 0} \zeta^{-n-1} \kappa^{n}$ in $\{\zeta ;|\zeta|=r\}$, the assertion follows easily. Moreover, by Proposition 1, the function $C[F]$ is a slice regular $\mathfrak{K}_{n}$-valued function in $U_{\sigma}$. Nevertheless, we are particularly interested in slice regular $\mathfrak{C}_{n}$-valued functions. 
The next result is a version of Theorem 4 from [18], stated in a quaternionic context.

Theorem 3. Let $U \subset \mathbb{C}$ be a conjugate symmetric open set and let $F \in \mathcal{O}\left(U, \mathfrak{K}_{n}\right)$. The Cauchy transform $C[F]$ is $\mathfrak{C}_{n}$-valued if and only if $F \in \mathcal{O}_{s}\left(U, \mathfrak{K}_{n}\right)$.

Proof. We first fix a paravector $\kappa \in U_{\sigma} \backslash \mathbb{R}$. If $\sigma(\kappa)=\left\{s_{+}, s_{-}\right\}$, the points $s_{+}, s_{-}$are distinct and not real. We then choose an $r>0$ sufficiently small such that, setting $D_{ \pm}:=\left\{\zeta \in U ;\left|\zeta-s_{ \pm}\right| \leq r\right\}$, we have $D_{ \pm} \subset U$ and $D_{+} \cap D_{-}=\emptyset$. Then

$$
C[F](\kappa)=\frac{1}{2 \pi i} \int_{\Gamma_{+}} F(\zeta)(\zeta-\kappa)^{-1} d \zeta+\frac{1}{2 \pi i} \int_{\Gamma_{-}} F(\zeta)(\zeta-\kappa)^{-1} d \zeta,
$$

where $\Gamma_{ \pm}$is the boundary of $D_{ \pm}$. We may write $F(\zeta)=\sum_{k \geq 0}\left(\zeta-s_{+}\right)^{k} a_{k}$ with $\zeta \in D_{+}, a_{k} \in \mathfrak{K}_{n}$ for all $k \geq 0$, as a uniformly convergent series. Similarly, $F(\zeta)=\sum_{k \geq 0}\left(\zeta-s_{-}\right)^{k} b_{k}$ with $\zeta \in D_{-}, b_{k} \in \mathfrak{K}_{n}$ for all $k \geq 0$, as a uniformly convergent series.

Note that

$$
\frac{1}{2 \pi i} \int_{\Gamma_{+}} F(\zeta)(\zeta-\kappa)^{-1} d \zeta=\sum_{k \geq 0}\left(a_{k} \frac{1}{2 \pi i} \int_{\Gamma_{+}}\left(\zeta-s_{+}\right)^{k}(\zeta-\kappa)^{-1} d \zeta\right)=a_{0} l_{+}\left(\mathfrak{s}_{\tilde{\kappa}}\right)
$$

because we have

$$
\frac{1}{2 \pi i} \int_{\Gamma_{+}}\left(\zeta-s_{+}\right)^{k}(\zeta-\kappa)^{-1} d \zeta=\left(\kappa-s_{+}\right)^{k} l_{+}\left(\mathfrak{s}_{\tilde{\kappa}}\right)
$$

by the analytic functional calculus of $\kappa$ (see also Lemma 1 ), which is equal to $l_{+}\left(\mathfrak{s}_{\tilde{\kappa}}\right)$ when $k=0$, and it is equal to 0 when $k \geq 1$, via the equality $\kappa l_{+}\left(\mathfrak{s}_{\tilde{\kappa}}\right)=s_{+} l_{+}\left(\mathfrak{s}_{\tilde{\kappa}}\right)$

Similarly

$$
\frac{1}{2 \pi i} \int_{\Gamma_{-}} F(\zeta)(\zeta-\kappa)^{-1} d \zeta=\sum_{k \geq 0}\left(b_{k} \frac{1}{2 \pi i} \int_{\Gamma_{-}}\left(\zeta-s_{-}\right)^{k}(\zeta-\kappa)^{-1} d \zeta\right)=b_{0} \iota_{-}\left(\mathfrak{s}_{\tilde{\kappa}}\right)
$$

because, as above, we have

$$
\left.\frac{1}{2 \pi i} \int_{\Gamma_{-}}\left(\zeta-s_{-}\right)^{k}(\zeta-\kappa)^{-1}\right) d \zeta=\left(\kappa-s_{-}\right)^{k} I_{-}\left(\mathfrak{s}_{\tilde{\kappa}}\right)
$$

which is equal $\iota_{-}\left(\mathfrak{s}_{\tilde{\kappa}}\right)$ when $k=0$, and it is equal to 0 when $k \geq 1$. Consequently,

$$
C[F](\kappa)=F\left(s_{+}\right) \iota_{+}\left(\mathfrak{s}_{\tilde{\kappa}}\right)+F\left(s_{-}\right) \iota_{-}\left(\mathfrak{s}_{\tilde{\kappa}}\right),
$$

and the right hand side of this equality coincides with the expression from formula (9).

Assume now that $\sigma(\kappa)=\{s\}$, where $s:=s_{+}=s_{-} \in \mathbb{R}$. Then necessarily $\kappa=s$, so fixing an $r>0$ such that the set $D:=\{\zeta \in U ;|\zeta-s| \leq r\} \subset U$, whose boundary is denoted by $\Gamma$, we have

$$
C[F](\kappa)=\frac{1}{2 \pi i} \int_{\Gamma} F(\zeta)(\zeta-\kappa)^{-1} d \zeta=F(s),
$$

via the usual analytic functional calculus.

In all of these situations, the element $C[F](\kappa)$ is equal to the right hand side of formula (9). Therefore, we must have $C[F](\kappa) \in \mathfrak{C}_{n}$ if and only if $F\left(s_{+}\right)=\overline{F\left(s_{-}\right)}$, via Theorem 1 . As every point $\lambda \in U$ is the eigenvalue of a certain paravector in $U_{\sigma}$, we deduce that $C[F](\kappa) \in \mathfrak{C}_{n}$ for all $\kappa \in U_{\sigma}$ if and only if $F: U \mapsto \mathfrak{K}_{n}$ is a stem function.

Remark 9. (1) It follows from the proof of the previous theorem that the element $C[F](\kappa)$, given by formula (11), coincides with the element $F_{\sigma}(\kappa)$ given by (9). To unify the notation, from now on this element will be denoted by $F_{\sigma}(\kappa)$, whenever $F$ is a stem function, analytic or not. 
(2) An important particular case is when let $f: U \mapsto \mathbb{C}$ is an analytic function, where $U \subset \mathbb{C}$ is a conjugate symmetric open set. In this case we may also consider the Cauchy transform of $f$ given by

$$
C[f](\kappa)=\frac{1}{2 \pi i} \int_{\Gamma} f(\zeta)(\zeta-\kappa)^{-1} d \zeta,
$$

where $\Gamma$ is the boundary of a Cauchy domain in $U$ containing the spectrum $\sigma(\kappa)$. According to Theorem 5, we have $C[f](\kappa) \in \mathfrak{C}_{n}$ if and only if $f$ is a stem function, that is $f \in \mathcal{O}_{s}(U)$. Of course, in this case we may (and shall) also use the notation $C[f]=f_{\sigma}$, and we have, in fact, $f_{\sigma} \in \mathcal{J F}\left(U_{\sigma}, \mathfrak{C}_{n}\right)$, by Corollary 2.

\section{Analytic Functional Calculus for Stem Functions}

Let $\Omega \subset \mathfrak{P}_{n}$ be a spectrally saturated open set, and let $U=\mathfrak{S}(\Omega) \subset \mathbb{C}$ (which is conjugate symmetric, and also open, by Remark 6(2)). We introduce the notation

$$
\begin{gathered}
\mathcal{R}_{s, n}(\Omega)=\left\{f_{\sigma} ; f \in \mathcal{O}_{s}(U)\right\}, \\
\mathcal{R}_{s}\left(\Omega, \mathfrak{C}_{n}\right)=\left\{F_{\sigma} ; F \in \mathcal{O}_{s}\left(U, \mathfrak{K}_{n}\right)\right\},
\end{gathered}
$$

which are $\mathbb{R}$-vector spaces.

In fact, these $\mathbb{R}$-vector spaces have some important properties, as already noticed in a quaternionic version of the next theorem (see Theorem 5 in [18]).

Theorem 4. Let $\Omega \subset \mathfrak{P}_{n}$ be a spectrally saturated open set, and let $U=\mathfrak{S}(\Omega)$. The space $\mathcal{R}_{s, n}(\Omega)$ is a unital commutative $\mathbb{R}$-algebra, the space $\mathcal{R}_{s}\left(\Omega, \mathfrak{C}_{n}\right)$ is a right $\mathcal{R}_{s, n}(\Omega)$-module, the linear map

$$
\mathcal{O}_{s}\left(U, \mathfrak{K}_{n}\right) \ni F \mapsto F_{\sigma} \in \mathcal{R}_{S}\left(\Omega, \mathfrak{C}_{n}\right)
$$

is a right module isomorphism, and its restriction

$$
\mathcal{O}_{s}(U) \ni f \mapsto f_{\sigma} \in \mathcal{R}_{s, n}(\Omega)
$$

is an $\mathbb{R}$-algebra isomorphism.

Moreover, for every polynomial $P(\zeta)=\sum_{j=0}^{m} a_{j} \zeta^{j}, \zeta \in \mathbb{C}$, with $a_{j} \in \mathfrak{C}_{n}$ for all $j=0,1, \ldots, m$, we have $P_{\sigma}(\kappa)=\sum_{j=0}^{m} a_{j} \kappa^{j} \in \mathfrak{C}_{n}$ for all $\kappa \in \mathfrak{P}_{n}$.

Proof. Thanks to Theorem 3, this statement is a particular case of Theorem 2. Indeed, the $\mathbb{R}$-linear maps

$$
\mathcal{O}_{s}\left(U, \mathfrak{K}_{n}\right) \ni F \mapsto F_{\sigma} \in \mathcal{R}_{s}\left(\Omega, \mathfrak{C}_{n}\right), \mathcal{O}_{s}(U) \ni f \mapsto f_{\sigma} \in \mathcal{R}_{s, n}(\Omega),
$$

are restrictions of the maps

$$
\mathcal{S}\left(U, \mathfrak{K}_{n}\right) \ni F \mapsto F_{\sigma} \in \mathcal{F}\left(\Omega, \mathfrak{C}_{n}\right), \mathcal{S}(U) \ni f \mapsto f_{\sigma} \in \mathcal{J F}\left(\Omega, \mathfrak{C}_{n}\right),
$$

respectively. Moreover, they are $\mathbb{R}$-isomorphisms, the latter being actually unital and multiplicative. Note that, in particular, for every polynomial $P(\zeta)=\sum_{j=0}^{m} a_{j} \zeta^{j}$ with $a_{j} \in \mathfrak{C}_{n}$ for all $j=0,1, \ldots, m$, we have $P_{\sigma}(\kappa)=$ $\sum_{j=0}^{m} a_{j} \kappa^{j} \in \mathfrak{C}_{n}$ for all $\kappa \in \mathfrak{P}_{n}$.

Remark 10. For every function $F \in \mathcal{O}_{s}\left(U, \mathfrak{K}_{n}\right)$, the derivatives $F^{(m)}$ also belong to $\mathcal{O}_{s}\left(U, \mathfrak{K}_{n}\right)$, where $U \subset \mathbb{C}$ is a conjugate symmetric open set.

Now fixing $F \in \mathcal{O}_{s}\left(U, \mathfrak{K}_{n}\right)$, we may define its extended derivatives with respect to the paravector variable via the formula

$$
F_{\sigma}^{(m)}(\kappa)=\frac{1}{2 \pi i} \int_{\Gamma} F^{(m)}(\zeta)(\zeta-\kappa)^{-1} d \zeta
$$


for the boundary $\Gamma$ of a Cauchy domain $\Delta \subset U, n \geq 0$ an arbitrary integer, and $\sigma(\kappa) \subset \Delta$.

In particular, if $\Delta$ is a disk centered at zero and $F \in \mathcal{O}_{s}\left(\Delta, \mathfrak{K}_{n}\right)$, so we have a representation of $F$ as a convergent series $\sum_{m \geq 0} a_{k} \zeta^{k}$ with coefficients in $\mathfrak{C}_{n}$, then (14) gives the equality $F_{\sigma}^{\prime}(\kappa)=\sum_{m \geq 1} m a_{m} \kappa^{m-1}$, which looks like a (formal) derivative of the function $F_{\sigma}(\kappa)=\sum_{m \geq 0} a_{k} \kappa^{m}$.

Remark 11. As already noticed in the framework of [18], Theorem 4 suggests a definition for $\mathfrak{C}_{n}$-valued "analytic functions" as elements of the set $\mathcal{R}_{s}\left(\Omega, \mathfrak{C}_{n}\right)$, where $\Omega$ is a spectrally saturated open subset of $\mathfrak{P}_{n}$. Because the expression "analytic function" is quite improper in this context, the elements of $\mathcal{R}_{s}\left(\Omega, \mathfrak{C}_{n}\right)$ will be called $\mathfrak{C}_{n}$-regular functions on $\Omega$. As shown by Theorem 4, the functions from $\mathcal{R}_{s}\left(\Omega, \mathfrak{C}_{n}\right)$ are the Cauchy transforms of the stem functions from $\mathcal{O}_{s}\left(U, \mathfrak{K}_{n}\right)$, with $U=\mathfrak{S}(\Omega)$.

Except for Theorem 4, many properties of $\mathfrak{C}_{n}$-regular functions can be obtained directly from the definition, by recapturing the corresponding results from [18]. We omit the details.

Remark 12. Let $U \subset \mathbb{C}$ be a conjugate symmetric open set, let $x, y \in \mathbb{R}$ with $y \neq 0$ and $z_{ \pm}=x \pm i y \in U$, let $F \in \mathcal{O}_{s}\left(U, \mathfrak{K}_{n}\right)$, and let $\mathfrak{s} \in \mathbb{S}_{n}$. We shall apply some arguments similar to those from Corollary 2, in a non commuting context.

Assuming $y>0$, we consider the paravectors $\kappa_{ \pm}=x \pm y_{\mathfrak{s}}$ for which $s_{+}\left(\kappa_{ \pm}\right)=x+i y, s_{-}\left(\kappa_{ \pm}\right)=x-i y$. As we have $\tilde{\kappa}_{ \pm}= \pm y \mathfrak{s}$, then $s_{\tilde{\kappa}_{ \pm}}= \pm \mathfrak{s}$, and $\iota_{ \pm}\left(s_{\tilde{\kappa}_{+}}\right)=(1 \mp i \mathfrak{s}) / 2, \iota_{ \pm}\left(s_{\tilde{\kappa}_{-}}\right)=(1 \pm i \mathfrak{s}) / 2$. Therefore,

$$
\begin{aligned}
& 2 F_{\sigma}\left(\kappa_{+}\right)=F\left(z_{+}\right)(1-i \mathfrak{s})+F\left(z_{-}\right)(1+i \mathfrak{s}), \\
& 2 F_{\sigma}\left(\kappa_{-}\right)=F\left(z_{+}\right)\left((1+i \mathfrak{s})+F\left(z_{-}\right)(1-i \mathfrak{s}) .\right.
\end{aligned}
$$

From these equations we deduce that

$$
\begin{aligned}
& 2 F\left(z_{+}\right)=F_{\sigma}\left(\kappa_{+}\right)(1-i \mathfrak{s})+F_{\sigma}\left(\kappa_{-}\right)(1+i \mathfrak{s}), \\
& 2 F\left(z_{-}\right)=F_{\sigma}\left(\kappa_{+}\right)(1+i \mathfrak{s})+F_{\sigma}\left(\kappa_{-}\right)(1-i \mathfrak{s}) .
\end{aligned}
$$

If $y<0$, for the paravectors $\kappa_{ \pm}=x \pm y_{\mathfrak{s}}$ we have $s_{+}\left(\kappa_{ \pm}\right)=x-i y, s_{-}\left(\kappa_{ \pm}\right)=$ $x+i y$. Moreover, as $\tilde{\kappa}_{ \pm}= \pm y \mathfrak{s}$, then $s_{\tilde{\kappa}_{ \pm}}=\mp \mathfrak{s}$, and $\iota_{ \pm}\left(s_{\tilde{\kappa}_{+}}\right)=(1 \pm i \mathfrak{s}) / 2$, $\iota_{ \pm}\left(s_{\tilde{\kappa}_{-}}\right)=(1 \mp i \mathfrak{s}) / 2$. Therefore

$$
\begin{aligned}
& 2 F_{\sigma}\left(\kappa_{+}\right)=F\left(z_{-}\right)(1+i \mathfrak{s})+F\left(z_{+}\right)(1-i \mathfrak{s}), \\
& 2 F_{\sigma}\left(\kappa_{-}\right)=F\left(z_{-}\right)\left((1-i \mathfrak{s})+F\left(z_{+}\right)(1+i \mathfrak{s}) .\right.
\end{aligned}
$$

These formulas lead again to equations (15) and (16). Consequently, we have the following (see also [2], Theorem 2.2.18 for a similar result, and [18] for the corresponding result in the quaternionic context).

The next proposition, and Remark 12 as well, have their counterparts in [18], stated as Proposition 3 and Remark 10, respectively. For a similar result see also Theorem 2.2.18 from [2].

Proposition 2. Let $U \subset \mathbb{C}$ be a conjugate symmetric open set, let $x, y \in \mathbb{R}$ with $x \pm i y \in U$, let $\mathfrak{s} \in \mathbb{S}_{n}$, and let $F \in \mathcal{O}_{s}\left(U, \mathfrak{K}_{n}\right)$. Then we have the formulas

$$
F(x \pm i y)=F_{\sigma}(x \pm y \mathfrak{s})\left(\frac{1 \mp i \mathfrak{s}}{2}\right)+F_{\sigma}(x \mp y \mathfrak{s})\left(\frac{1 \pm i \mathfrak{s}}{2}\right) .
$$

As the proof has been previously done, we only note that equality (17) also holds for $y=0$.

Lemma 3. Let $U \subset \mathbb{C}$ be a conjugate symmetric open set, let $\mathfrak{s} \in \mathbb{S}_{n}$ be fixed, and let $\Psi: U_{\mathfrak{s}} \mapsto \mathfrak{C}_{n}$ be such that $\bar{\partial}_{ \pm \mathfrak{s}} \Psi=0$. Then there exists a function $\Phi \in \mathcal{R}_{\mathfrak{s}}\left(U_{\sigma}, \mathfrak{C}_{n}\right)$ with $\Psi=\Phi \mid U_{\mathfrak{s}}$, where $U_{\mathfrak{s}}=\{x+y \mathfrak{s} ; x+i y \in U\}$.

Proof. For arbitrary points $z_{ \pm}=x \pm i y \in U$ with $x, y(\neq 0) \in \mathbb{R}$, as in Remark 12, we consider the paravectors $\kappa_{ \pm}=x \pm y \mathfrak{s}$, so $s_{+}\left(\kappa_{ \pm}\right)=x+i|y|$, and $s_{-}\left(\kappa_{ \pm}\right)=x-i|y|$. Inspired by formula (17), we set

$$
2 F\left(z_{+}\right)=\Psi\left(\kappa_{+}\right)(1-i \mathfrak{s})+\Psi\left(\kappa_{-}\right)(1+i \mathfrak{s}),
$$




$$
2 F\left(z_{-}\right)=\Psi\left(\kappa_{+}\right)(1+i \mathfrak{s})+\Psi\left(\kappa_{-}\right)(1-i \mathfrak{s})
$$

Then we have

$$
2 \frac{\partial F\left(z_{+}\right)}{\partial x}=\frac{\partial \Psi\left(\kappa_{+}\right)}{\partial x}(1-i \mathfrak{s})+\frac{\partial \Psi\left(\kappa_{-}\right)}{\partial x}(1+i \mathfrak{s}),
$$

and

$$
2 i \frac{\partial F\left(z_{+}\right)}{\partial y}=\frac{\partial \Psi\left(\kappa_{+}\right)}{\partial y} \mathfrak{s}(1-i \mathfrak{s})+\frac{\partial \Psi\left(\kappa_{-}\right)}{\partial y}(-\mathfrak{s})(1+i \mathfrak{s}),
$$

because $i(1-i \mathfrak{s})=\mathfrak{s}(1-i \mathfrak{s})$ and $i(1+i \mathfrak{s})=-\mathfrak{s}(1+i \mathfrak{s})$.

Therefore,

$$
\frac{\partial F\left(z_{+}\right)}{\partial x}+i \frac{\partial F\left(z_{+}\right)}{\partial y}=\left(\bar{\partial}_{\mathfrak{s}} \Psi\left(\kappa_{+}\right)\right)(1-i \mathfrak{s})+\left(\bar{\partial}_{-\mathfrak{s}} \Psi\left(\kappa_{-}\right)\right)(1+i \mathfrak{s})=0,
$$

showing that the function $z_{+} \mapsto F\left(z_{+}\right)$is analytic in $U$.

Because $\overline{F\left(z_{-}\right)}=\overline{F\left(\overline{\left.z_{+}\right)}\right.}=F\left(z_{+}\right)$, and when $y=0$ we have $\overline{F\left(z_{-}\right)}=F\left(z_{+}\right)=F(x)$, we have constructed a function $F \in \mathcal{O}_{s}\left(U, \mathfrak{K}_{n}\right)$. Hence, taking $\Phi=F_{\sigma}$, we have $\Phi \in \mathcal{R}_{s}\left(U_{\sigma}, \mathfrak{C}_{n}\right)$ with $\Psi=\Phi \mid U_{\mathfrak{s}}$, via Remark 12 .

The next theorem is a version of Theorem 6 from [18].

Theorem 5. Let $\Omega \subset \mathfrak{P}_{n}$ be a spectrally saturated open set, and let $\Phi: \Omega \mapsto \mathfrak{C}_{n}$. The following conditions are equivalent:

(i) $\Phi$ is a slice regular function;

(ii) $\Phi \in \mathcal{R}_{s}\left(\Omega, \mathfrak{C}_{n}\right)$, that is, $\Phi$ is $\mathfrak{C}_{n}$-regular.

Proof. If $\Phi \in \mathcal{R}_{s}\left(\Omega, \mathfrak{C}_{n}\right)$, then $\Phi$ is slice regular, by Proposition 1, so (ii) $\Rightarrow$ (i).

Conversely, let $\Phi$ be slice regular in $\Omega$. Fixing an $\mathfrak{s} \in \mathbb{S}_{n}$, we have $\bar{\partial}_{ \pm \mathfrak{s}} \Phi_{\mathfrak{s}}=0$, where $\Phi_{\mathfrak{s}}=\Phi \mid U_{\mathfrak{s}}$. It follows from Lemma 3 that there exists $\Psi \in \mathcal{R}\left(U_{\sigma}, \mathfrak{C}_{n}\right)$ with $\Psi_{\mathfrak{s}}=\Phi_{\mathfrak{s}}$. This implies that $\Phi=\Psi$, because both $\Phi, \Psi$ are uniquely determined by $\Phi_{\mathfrak{s}}, \Psi_{\mathfrak{s}}$, respectively, the former by (the right hand version of) Lemma 2.2.24 in [2], and the latter by Remark 7 . Consequently, we also have $(i) \Rightarrow$ (ii).

Remark A concept of "Cliffordian holomorphic function" also appears in [11], in a different context.

\section{Spectrum of Clifford Operators}

Let $\mathcal{V}$ be a Banach $\mathrm{Cl}$-space, and let $\mathcal{V}_{\mathbb{C}}=\mathcal{V}+i \mathcal{V}$ its complexification, endoved with the norm $\|x+i y\|=$ $\|x\|+\|y\|$, for all $x, y \in \mathcal{V}$, where $\left\|{ }^{\star}\right\|$ is the norm of $\mathcal{V}$. We denote by $C$ the conjugation on $\mathcal{V}_{\mathbb{C}}$, that is, the map $C(x+i y)=x-i y$ for all $x, y \in \mathcal{V}$, which is an $\mathbb{R}$-linear map whose square is the identity.

As in Subsection 2.3, for every $T \in \mathcal{B}(\mathcal{V})$, we consider its natural "complex extension" to $\mathcal{V}_{\mathbb{C}}$ given by $T_{\mathbb{C}}(x+i y)=T x+i T y$, for all $x, y \in \mathcal{V}$, which is at least $\mathbb{C}$-linear (if $T \in \mathcal{B}^{\mathrm{r}}(\mathcal{V}), T_{\mathbb{C}}$ is right $\mathfrak{K}_{n}$-linear), so $T_{\mathbb{C}} \in \mathcal{B}\left(\mathcal{V}_{\mathbb{C}}\right)$. As already noticed, the map $\mathcal{B}(\mathcal{V}) \ni T \mapsto T_{\mathbb{C}} \in \mathcal{B}\left(\mathcal{V}_{\mathbb{C}}\right)$ is a unital injective morphism of real algebras.

Assuming that $\mathcal{V}$ is a Banach $\mathrm{Cl}$-space implies that $\mathcal{B}^{\mathrm{r}}(\mathcal{V})$ is a unital real Banach $\mathrm{Cl}$-algebra (that is, a Banach algebra which also a Banach $C l$-space), via the algebraic operations $(\mathbf{a} T)(x)=\mathbf{a} T(x)$, and $(T \mathbf{a})(x)=$ $T(\mathbf{a} x)$ for all $\mathbf{a} \in \mathfrak{C}_{n}$ and $x \in \mathcal{V}$. The complexification $\mathcal{B}^{\mathrm{r}}(\mathcal{V})_{\mathbb{C}}$ of $\mathcal{B}^{\mathrm{r}}(\mathcal{V})$ is, in particular, a unital complex Banach algebra, with the product

$$
\left(T_{1}+i T_{2}\right)\left(S_{1}+i S_{2}\right)=T_{1} S_{1}-T_{2} S_{2}+i\left(T_{1} S_{2}+T_{2} S_{1}\right), T_{1}, T_{2}, S_{1}, S_{2} \in \mathcal{B}^{\mathrm{r}}(\mathcal{V}),
$$
and a fixed norm, say $\left\|\left(T_{1}+i T_{2}\right)\right\|=\left\|T_{1}\right\|+\left\|T_{2}\right\|, T_{1}, T_{2} \in \mathcal{B}^{\mathrm{r}}(\mathcal{V})$.

Also note that the complex numbers, regarded as elements of $\mathcal{B}^{r}(\mathcal{V})_{\mathbb{C}}$, commute with the elements of $\mathcal{B}^{\mathrm{r}}(\mathcal{V})$.

Remark 13. For every $S \in \mathcal{B}\left(\mathcal{V}_{\mathbb{C}}\right)$ we put $S^{b}=\operatorname{CSC} \in \mathcal{B}\left(\mathcal{V}_{\mathbb{C}}\right)$. The assignment $S \mapsto S^{b}$ is a conjugate linear automorphism of the algebra $\mathcal{B}\left(\mathcal{V}_{\mathbb{C}}\right)$, whose square is the identity operator. In fact, the map $S \mapsto S^{b}$ is a conjugation of $\mathcal{B}\left(\mathcal{V}_{\mathbb{C}}\right)$, induced by $C$. Moreover, $S^{b}=S$ if and only if $S(\mathcal{V}) \subset \mathcal{V}$. In particular, we have $S=$ 
$S_{1}+i S_{2}$ with $S_{j}(\mathcal{V}) \subset \mathcal{V}, j=1,2$, uniquely determined. Its action on the space $\mathcal{V}_{\mathbb{C}}$ is given by $S(x+i y)=$ $S_{1} x-S_{2} y+i\left(S_{1} y+S_{2} x\right)$ for all $x, y \in \mathcal{V}$.

Because $C R_{\mathbf{a}}=R_{\mathbf{a}} C$ for all $\mathbf{a} \in \mathfrak{P}_{n}$, it follows that if $S \in \mathcal{B}^{\mathrm{r}}\left(\mathcal{V}_{\mathbb{C}}\right)$, then $S^{b} \in \mathcal{B}^{\mathrm{r}}\left(\mathcal{V}_{\mathbb{C}}\right)$. In particular, we have $\left(S+S^{b}\right)(\mathcal{V}) \subset \mathcal{V}, i\left(S-S^{b}\right)(\mathcal{V}) \subset \mathcal{V}$, and $\left(T_{\mathbb{C}}\right)^{b}=T_{\mathbb{C}}$ for all $T \in \mathcal{B}^{\mathrm{r}}(\mathcal{V})$. Note also that the map

$$
\mathcal{B}^{\mathrm{r}}(\mathcal{V}) \ni T \mapsto T_{\mathbb{C}} \in\left\{S=S^{\mathrm{b}} ; S \in \mathcal{B}^{\mathrm{r}}\left(\mathcal{V}_{\mathbb{C}}\right)\right\}
$$

is actually a real unital algebra isomorphism, since its surjectivity follows from the equality $(S \mid \mathcal{V})_{\mathbb{C}}=S$ whenever $S=S^{b} \in \mathcal{B}^{r}\left(\mathcal{V}_{\mathbb{C}}\right)$. This implies that the algebras $\mathcal{B}^{\mathrm{r}}\left(\mathcal{V}_{\mathbb{C}}\right)$ and $\mathcal{B}^{\mathrm{r}}(\mathcal{V})_{\mathbb{C}}$ are isomorphic. This isomorphism is given by the assignment

$$
\mathcal{B}^{\mathrm{r}}(\mathcal{V})_{\mathbb{C}} \ni T_{1}+i T_{2} \mapsto T_{1 \mathbb{C}}+i T_{2 \mathbb{C}} \in \mathcal{B}^{\mathrm{r}}\left(\mathcal{V}_{\mathbb{C}}\right)
$$

which is is actually an algebra isomorphism, via a direct calculation.

The continuity of this assignment is also clear, and therefore it is a Banach algebra isomorphism. For this reason, may identify the algebras $\mathcal{B}^{r}\left(\mathcal{V}_{\mathbb{C}}\right)$ and $\mathcal{B}^{r}(\mathcal{V})_{\mathbb{C}}$. As already noticed above, the real algebras $\mathcal{B}^{r}(\mathcal{V})$ and $\left\{S \in \mathcal{B}^{\mathrm{r}}\left(\mathcal{V}_{\mathbb{C}}\right) ; S=S^{b}\right\}$ may and will be also identified.

The operators from the algebra $\mathcal{B}^{\mathrm{r}}(\mathcal{V})$ will be sometimes called Clifford operators, or simply $\mathrm{Cl}$-operators.

Looking at Definition 3.1.4 from [2], we can give the folowing.

Definition 6. For a given operator $T \in B^{\mathrm{r}}(\mathcal{V})$, the set

$$
\left.\sigma_{C l}(T):=\left\{\kappa \in \mathfrak{P}_{n} ; T^{2}-2 \Re(\kappa) T+|\kappa|^{2}\right) \text { not invertible }\right\}
$$

is called the Clifford (or $\mathrm{Cl}$-)spectrum of $\mathrm{T}$.

The complement $\rho_{C l}(T)=\mathfrak{P}_{n} \backslash \sigma_{C l}(T)$ is called the Clifford (or $\mathrm{Cl}$-)resolvent of $T$.

Note that, if $\mathbf{a} \in \sigma_{C l}(T)$, then $\left\{\mathbf{b} \in \mathfrak{P}_{n} ; \sigma(\mathbf{b})=\sigma(\mathbf{a})\right\} \subset \sigma_{C l}(T)$.

Since every operator $T \in \mathcal{B}^{\mathrm{r}}(\mathcal{V})$ is, in particular, $\mathbb{R}$-linear, we also have a complex resolvent, defined by

$$
\begin{gathered}
\rho_{\mathbb{C}}(T)=\left\{\lambda \in \mathbb{C} ;\left(T^{2}-2 \Re(\lambda) T+|\lambda|^{2}\right)^{-1} \in \mathcal{B}^{\mathrm{r}}(\mathcal{V})\right\}= \\
\left\{\lambda \in \mathbb{C} ;\left(\lambda-T_{\mathbb{C}}\right)^{-1} \in \mathcal{B}^{\mathrm{r}}\left(\mathcal{V}_{\mathbb{C}}\right)\right\}=\rho\left(T_{\mathbb{C}}\right),
\end{gathered}
$$

and the associated complex spectrum $\sigma_{\mathbb{C}}(T)=\sigma\left(T_{\mathbb{C}}\right)$ as well.

Note that both sets $\sigma_{\mathbb{C}}(T)$ and $\rho_{\mathbb{C}}(T)$ are conjugate symmetric.

There exists a strong connexion between $\sigma_{C l}(T)$ and $\sigma_{\mathbb{C}}(T)$. In fact, the set $\sigma_{\mathbb{C}}(T)$ looks like a "complex border" of the set $\sigma_{C l}(T)$. Specifically, we can prove the following.

Lemma 4. For every $T \in \mathcal{B}^{\mathrm{r}}(\mathcal{V})$ we have the equalities

$$
\sigma_{C l}(T)=\left\{\kappa \in \mathfrak{P}_{n} ; \sigma_{\mathbb{C}}(T) \cap \sigma(\kappa) \neq \emptyset\right\} .
$$

and

$$
\sigma_{\mathbb{C}}(T)=\left\{\lambda \in \sigma(\kappa) ; \kappa \in \sigma_{C l}(T)\right\}
$$

Proof. Let us prove (18). If $\kappa \in \sigma_{C l}(T)$, and so the $T^{2}-2 \Re(\kappa) T+|\kappa|^{2}$ is not invertible, choosing $\lambda \in\{\Re(\kappa) \pm$ $i|\Im(\kappa)|\}=\sigma(\kappa)$, we clearly have $T^{2}-2 \Re(\lambda) T+|\lambda|^{2}$ not invertible, implying $\lambda \in \sigma_{\mathbb{C}}(T) \cap \sigma(\kappa) \neq \emptyset$.

Conversely, if for some $\kappa \in \mathfrak{P}_{n}$ there exists $\lambda \in \sigma_{\mathbb{C}}(T) \cap \sigma(\kappa)$, and so $T^{2}-2 \Re(\lambda) T+|\lambda|^{2}=T^{2}-2 \Re(\kappa) T+|\kappa|^{2}$ is not invertible, we must have $\kappa \in \sigma_{C l}(T)$.

We now prove (19). Let $\lambda \in \sigma_{\mathbb{C}}(T)$, so the operator $T^{2}-2(\Re \lambda) T+|\lambda|^{2}$ is not invertible. Setting $\kappa=\Re(\lambda)+$ $|\Im \lambda| \mathfrak{s}$, with $\mathfrak{s} \in \mathbb{S}_{n}$, we have $\lambda \in \sigma(\kappa)$. Moreover, $T^{2}+2 \Re(\kappa) T+|\kappa|^{2}$ is not invertible, and so $\kappa \in \sigma_{C l}(T)$.

Conversely, if $\lambda \in \sigma(\kappa)$ for some $\kappa \in \sigma_{C l}(T)$, then $\lambda \in\{\Re(\kappa) \pm i|\Im(\kappa)|\}$, showing that $T^{2}-2 \Re(\lambda) T+|\lambda|^{2}=$ $T^{2}+2 \Re(\kappa) T+|\kappa|^{2}$ is not invertible. 
Remark 14. As expected, the set $\sigma_{C l}(T)$ is nonempty and bounded, which follows from Lemma 4. In fact, we have the equality

$$
\sigma_{C l}(T)=\left\{\Re(\lambda)+|\Im(\lambda)| \mathfrak{s} ; \lambda \in \sigma_{\mathbb{C}}(T), \mathfrak{s} \in \mathbb{S}_{n}\right\} .
$$

It is also closed, as a consequence of Definition 6, because the set of invertible elements in $\mathcal{B}^{r}(\mathcal{V})$ is open.

Note that the subset $\sigma_{C l}(T)$ is spectrally saturated (see Definition 2(2)).

\section{Analytic Functional Calculus for Clifford Operators}

Having a concept of spectrum for Clifford operators, an important step for further development is the construction of an analytic functional calculus. We follow the main ideas from [19].

If $\mathcal{V}$ is a Banach $\mathrm{Cl}$-space, and so each operator $T \in \mathcal{B}^{\mathrm{r}}(\mathcal{V})$ has a complex spectrum $\sigma_{\mathbb{C}}(T)$, one can use the classical Riesz-Dunford functional calculus, in a slightly generalized form (that is, replacing the scalar-valued analytic functions by operator-valued analytic ones, which is a well known idea).

Remark 15. If $\mathcal{V}$ is Banach $\mathrm{Cl}$-space, and $T \in \mathcal{B}(\mathcal{V})$, we have the usual analytic functional calculus for the operator $T_{\mathbb{C}} \in \mathcal{B}\left(\mathcal{V}_{\mathbb{C}}\right)$ (see [4]). That is, in a slightly generalized form, and for later use, if $U \supset \sigma\left(T_{\mathbb{C}}\right)$ is an open set in $\mathbb{C}$ and $F: U \mapsto B\left(\mathcal{V}_{\mathbb{C}}\right)$ is analytic, the (left) Riesz-Dunford analytic functional calculus is given by the formula

$$
F\left(T_{\mathbb{C}}\right)=\frac{1}{2 \pi i} \int_{\Gamma} F(\zeta)\left(\zeta-T_{\mathbb{C}}\right)^{-1} d \zeta,
$$

where $\Gamma$ is the boundary of a Cauchy domain $\Delta$ containing $\sigma\left(T_{\mathbb{C}}\right)$ in $U$. In fact, since $\sigma\left(T_{\mathbb{C}}\right)$ is conjugate symmetric, we may and shall assume that both $U$ and $\Gamma$ are conjugate symmetric. Because the function $\zeta \mapsto F(\zeta)\left(\zeta-T_{\mathbb{C}}\right)^{-1}$ is analytic in $U \backslash \sigma\left(T_{\mathbb{C}}\right)$, the integral does not depend on the particular choice of the Cauchy domain $\Delta$ containing $\sigma\left(T_{\mathbb{C}}\right)$.

A natural question is to find an appropriate condition to have $F\left(T_{\mathbb{C}}\right)^{b}=F\left(T_{\mathbb{C}}\right)$, which would imply the invariance of $\mathcal{V}$ under $F\left(T_{\mathbb{C}}\right)$.

Remark 16. If $\mathcal{A}$ is a unital real Banach algebra, $\mathcal{A}_{\mathbb{C}}$ its complexification, and $U \subset \mathbb{C}$ is open, we denote by $\mathcal{O}\left(U, \mathcal{A}_{\mathbb{C}}\right)$ the algebra of all analytic $\mathcal{A}_{\mathbb{C}}$-valued functions. If $U$ is conjugate symmetric, and $\mathcal{A}_{\mathbb{C}} \ni a \mapsto \bar{a} \in$ $\mathcal{A}_{\mathbb{C}}$ is its natural conjugation, we denote by $\mathcal{O}_{s}\left(U, \mathcal{A}_{\mathbb{C}}\right)$ the real subalgebra of $\mathcal{O}\left(U, \mathcal{A}_{\mathbb{C}}\right)$ consisting of those functions $F$ with the property $F(\bar{\zeta})=\overline{F(\zeta)}$ for all $\zeta \in U$. As in Definition 3, such functions will be called $\left(\mathcal{A}_{\mathbb{C}}\right.$-valued $)$ stem functions.

When $\mathcal{A}=\mathbb{R}$, so $\mathcal{A}_{\mathbb{C}}=\mathbb{C}$, the space $\mathcal{O}_{s}(U, \mathbb{C})$ will be denoted by $\mathcal{O}_{s}(U)$, which is a real algebra. Note that $\mathcal{O}_{s}\left(U, \mathcal{A}_{\mathbb{C}}\right)$ is also a two-sided $\mathcal{O}_{s}(U)$-module.

With the notation of Remark 15, we state and prove the following adapted version of Theorem 1 from [19].

Theorem 6. Let $U \subset \mathbb{C}$ be open and conjugate symmetric. If $F \in \mathcal{O}_{S}\left(U, \mathcal{B}\left(\mathcal{V}_{\mathbb{C}}\right)\right)$, we have $F\left(T_{\mathbb{C}}\right)^{b}=F\left(T_{\mathbb{C}}\right)$ for all $T \in \mathcal{B}(\mathcal{V})$ with $\sigma_{\mathbb{C}}(T) \subset U$.

Moreover, if $F \in \mathcal{O}_{s}\left(U, \mathcal{B}^{\mathrm{r}}\left(\mathcal{V}_{\mathbb{C}}\right)\right)$, and $T \in \mathcal{B}^{\mathrm{r}}(\mathcal{V})$, then $F\left(T_{\mathbb{C}}\right) \in \mathcal{B}^{\mathrm{r}}\left(\mathcal{V}_{\mathbb{C}}\right)$.

Proof. We use the notation from Remark 15, ssuming $\Gamma$ conjugate symmetric. We put $\Gamma_{ \pm}:=\Gamma \cap \mathbb{C}_{ \pm}$, where $\mathbb{C}_{+}$(resp. $\mathbb{C}_{-}$) equals to $\{\lambda \in \mathbb{C} ; \Im \lambda \geq 0\}$ (resp. $\{\lambda \in \mathbb{C} ; \Im \lambda \leq 0\}$ ). We write $\Gamma_{+}=\cup_{j=1}^{m} \Gamma_{j+}$, where $\Gamma_{j+}$ are the connected components of $\Gamma_{+}$. Similarly, we write $\Gamma_{-}=\cup_{j=1}^{m} \Gamma_{j-}$, where $\Gamma_{j-}$ are the connected components of $\Gamma_{-}$, and $\Gamma_{j-}$ is the reflexion of $\Gamma_{j+}$ with respect of the real axis.

As $\Gamma$ is a finite union of Jordan piecewise smooth closed curves, for each index $j$ we have a parametrization $\phi_{j}:[0,1] \mapsto \mathbb{C}$, positively oriented, such that $\phi_{j}([0,1])=\Gamma_{j+}$. Taking into account that the function $t \mapsto \overline{\phi_{j}(t)}$ 
is a parametrization of $\Gamma_{j-}$ negatively oriented, and setting $\Gamma_{j}=\Gamma_{j+} \cup \Gamma_{j_{-}}$, we can write

$$
\begin{aligned}
& F_{j}\left(T_{\mathbb{C}}\right):=\frac{1}{2 \pi i} \int_{\Gamma_{j}} F(\zeta)\left(\zeta-T_{\mathbb{C}}\right)^{-1} d \zeta= \\
& \frac{1}{2 \pi i} \int_{0}^{1} F\left(\phi_{j}(t)\right)\left(\phi_{j}(t)-T_{\mathbb{C}}\right)^{-1} \phi_{j}^{\prime}(t) d t \\
& -\frac{1}{2 \pi i} \int_{0}^{1} F\left(\overline{\phi_{j}(t)}\right)\left(\overline{\phi_{j}(t)}-T_{\mathbb{C}}\right)^{-1} \overline{\phi_{j}^{\prime}(t)} d t .
\end{aligned}
$$

Therefore,

$$
\begin{gathered}
F_{j}\left(T_{\mathbb{C}}\right)^{b}=-\frac{1}{2 \pi i} \int_{0}^{1} F\left(\phi_{j}(t)\right)^{b}\left(\overline{\phi_{j}(t)}-T_{\mathbb{C}}\right)^{-1} \overline{\phi_{j}^{\prime}(t)} d t \\
+\frac{1}{2 \pi i} \int_{0}^{1} F\left(\overline{\phi_{j}(t)}\right)^{b}\left(\phi_{j}(t)-T_{\mathbb{C}}\right)^{-1} \phi_{j}^{\prime}(t) d t .
\end{gathered}
$$

According to our assumption on the function $F$, we obtain $F_{j}\left(T_{\mathbb{C}}\right)=F_{j}\left(T_{\mathbb{C}}\right)^{b}$ for all $j$, and therefore

$$
F\left(T_{\mathbb{C}}\right)^{b}=\sum_{j=1}^{m} F_{j}\left(T_{\mathbb{C}}\right)^{b}=\sum_{j=1}^{m} F_{j}\left(T_{\mathbb{C}}\right)=F\left(T_{\mathbb{C}}\right) .
$$

Moreover, if $T \in \mathcal{B}^{\mathrm{r}}(\mathcal{V})$, because $R_{\mathbf{a}} F(\zeta)\left(\zeta-T_{\mathbb{C}}\right)^{-1}=F(\zeta)\left(\zeta-T_{\mathbb{C}}\right)^{-1} R_{\mathbf{a}}$ for all $\mathbf{a} \in \mathfrak{P}_{n}$, via the definition of $\mathcal{B}^{\mathrm{r}}\left(\mathcal{V}_{\mathbb{C}}\right)$, it follows that $F\left(T_{\mathbb{C}}\right) \in \mathcal{B}^{\mathrm{r}}\left(\mathcal{V}_{\mathbb{C}}\right)$.

In the next result, we identify the algebra $\mathcal{B}^{\mathbf{r}}(\mathcal{V})$ with a real subalgebra of $\mathcal{B}^{\mathbf{r}}(\mathcal{V})_{\mathbb{C}}$, in turn identified with $\mathcal{B}^{\mathbf{r}}\left(\mathcal{V}_{\mathbb{C}}\right)$ (see Remark 13). In this case, when $F \in \mathcal{O}_{s}\left(U, \mathcal{B}^{\mathbf{r}}(\mathcal{V})_{\mathbb{C}}\right)$, we shall write

$$
F(T)=\frac{1}{2 \pi i} \int_{\Gamma} F(\zeta)(\zeta-T)^{-1} d \zeta,
$$

noting that the right hand side of this formula belongs to $\mathcal{B}^{r}(\mathcal{V})$, as a consequence of Theorem 6 .

The following result expresses the (left) analytic functional calculus of a given operator from $\mathcal{B}^{\mathrm{r}}(\mathcal{V})$ with $\mathcal{B}^{r}(\mathcal{V})_{\mathbb{C}}$-valued stem functions. It is a version of Theorem 4 from [19], proved in a quaternionic context.

Theorem 7. Let $\mathcal{V}$ be a Banach $C l$-space, let $U \subset \mathbb{C}$ be a conjugate symmetric open set, and let $T \in \mathcal{B}^{\mathrm{r}}(\mathcal{V})$, with $\sigma_{\mathbb{C}}(T) \subset U$. Then the assignment

$$
\mathcal{O}_{s}\left(U, \mathcal{B}^{\mathrm{r}}(\mathcal{V})_{\mathbb{C}}\right) \ni F \mapsto F(T) \in \mathcal{B}^{\mathrm{r}}(\mathcal{V})
$$

is an $\mathbb{R}$-linear map, and the map

$$
\mathcal{O}_{s}(U) \ni f \mapsto f(T) \in \mathcal{B}^{\mathrm{r}}(\mathcal{V})
$$

is a unital real algebra morphism.

Moreover, the following properties hold true:

(1) for all $F \in \mathcal{O}_{S}\left(U, \mathcal{B}^{\mathbf{r}}(\mathcal{V})_{\mathbb{C}}\right), f \in \mathcal{O}_{S}(U)$, we have $(F f)(T)=F(T) f(T)$.

(2) for every polynomial $P(\zeta)=\sum_{j=0}^{m} A_{j} \zeta^{j}, \zeta \in \mathbb{C}$, with $A_{j} \in \mathcal{B}^{\mathbf{r}}(\mathcal{V})$ for all $j=0,1, \ldots, m$, we have $P(T)=$ $\sum_{j=0}^{m} A_{j} T^{j} \in \mathcal{B}^{\mathbf{r}}(\mathcal{V})$

Proof. The arguments are more or less standard (see [4]). The $\mathbb{R}$-linearity of the maps

$$
\mathcal{O}_{s}\left(U, \mathcal{B}^{\mathrm{r}}(\mathcal{V})_{\mathbb{C}}\right) \ni F \mapsto F(T) \in \mathcal{B}^{\mathrm{r}}(\mathcal{V}), \mathcal{O}_{s}(U) \ni f \mapsto f(T) \in \mathcal{B}^{\mathrm{r}}(\mathcal{V}),
$$


is clear. The second one is actually (unital and) multiplicative, which follows from the multiplicativiry of the usual analytic functional calculus of $T$.

In fact, we have $(F f)(T)=F(T) f(T)$ for all $F \in \mathcal{O}_{S}\left(U, \mathcal{B}^{\mathbf{r}}(\mathcal{V})_{\mathbb{C}}\right), f \in \mathcal{O}_{S}(U)$. This follows from the equalities,

$$
\begin{gathered}
(F f)(T)=\frac{1}{2 \pi i} \int_{\Gamma_{0}} F(\zeta) f(\zeta)(\zeta-T)^{-1} d \zeta= \\
\left(\frac{1}{2 \pi i} \int_{\Gamma_{0}} F(\zeta)(\zeta-T)^{-1} d \zeta\right)\left(\frac{1}{2 \pi i} \int_{\Gamma} f(\eta)(\eta-T)^{-1} d \eta\right)=F(T) f(T),
\end{gathered}
$$

obtained as in the classical case (see [4], Section VII.3), holding because $f$ is $\mathbb{C}$-valued and commutes with the operators in $\mathcal{B}^{\mathbf{r}}(\mathcal{V})$. Here $\Gamma, \Gamma_{0}$ are the boundaries of two Cauchy domains $\Delta, \Delta_{0}$ respectively, such that $\Delta \supset \bar{\Delta}_{0}$, and $\Delta_{0}$ contains $\sigma_{\mathbb{C}}(T)$.

Note that, in particular, for every polynomial $P(\zeta)=\sum_{j=0}^{m} A_{j} \zeta^{j}$ with $A_{j} \in \mathcal{B}^{\mathrm{r}}(\mathcal{V})$ for all $j=0,1, \ldots, m$, we have $P(T)=\sum_{j=0}^{m} A_{j} T^{j} \in \mathcal{B}^{\mathbf{r}}(\mathcal{V})$ for all $T \in \mathcal{B}^{\mathbf{r}}(\mathcal{V})$.

Corollary 3. Let $\mathcal{V}$ be a Banach $\mathrm{Cl}$-space, let $U \subset \mathbb{C}$ be a conjugate symmetric open set, and let $T \in \mathcal{B}^{\mathrm{r}}(\mathcal{V})$, with $\sigma_{\mathbb{C}}(T) \subset U$. There exists an assignment

$$
\mathcal{O}_{s}\left(U, \mathfrak{K}_{n}\right) \ni F \mapsto F(T) \in \mathcal{B}^{\mathrm{r}}(\mathcal{V}),
$$

which is an $\mathbb{R}$-linear map, such that

(1) for all $F \in \mathcal{O}_{s}\left(U, \mathfrak{K}_{n}\right), f \in \mathcal{O}_{s}(U)$, we have $(F f)(T)=F(T) f(T)$.

(2) for every polynomial $P(\zeta)=\sum_{j=0}^{m} \mathbf{a}_{j} \zeta^{j}, \zeta \in \mathbb{C}$, with $\mathbf{a}_{j} \in \mathfrak{C}_{n}$ for all $j=0,1, \ldots, m$, we have $P(T)=$ $\sum_{j=0}^{m} \mathbf{a}_{j} T^{j} \in \mathcal{B}^{\mathbf{r}}(\mathcal{V})$.

Proof. Note that the algebra $\mathcal{O}_{s}\left(U, \mathfrak{K}_{n}\right)$, can be regarded as a subalgebra of the algebra $\mathcal{O}_{s}\left(U, \mathcal{B}^{r}(V)_{\mathbb{C}}\right)$, whose elements are identified with left multiplication operators. Therefore, this corollary is a direct consequence of Theorem 7.

Remark 17. The space $\mathcal{R}_{S}\left(\Omega, \mathfrak{C}_{n}\right)$, introduced in Section 6 , can be independently defined, and it consists of the set of all $\mathfrak{C}_{n}$-valued functions, which are slice monogenic in the sense of [2], Definition 2.2.2 (or slice regular, as called in this work). They are used in [2] to define a functional calculus for tuples of not necessarily commuting real linear operators. Specifically, with a slightly modified notation, given an arbitrary family $\left(T_{0}, T_{1}, \ldots, T_{n}\right)$, acting on the real space $\mathcal{V}$, it is associated with the operator $\mathbf{T}=\sum_{j=0}^{n} T_{j} \otimes e_{j}$, acting on the two-sided $\mathfrak{C}_{n}$-module $\mathcal{V}_{n}=\mathcal{V} \otimes_{\mathbb{R}} \mathfrak{C}_{n}$. In fact, the symbol " $\otimes$ " may (and will) be omitted. Moreover, as alluded in [2], page 83, we may work on a Banach $\mathrm{Cl}$ - $\operatorname{space} \mathcal{V}$, and using operators from $\mathcal{B}^{\mathrm{r}}(\mathcal{V})$.

Roughly speaking, after fixing a Clifford operator, each regular $\mathfrak{C}_{n}$-valued function defined in a neighborhood $\Omega$ of its $\mathrm{Cl}$-spectrum is associated with another Clifford operator, replacing formally the paravector variable with that operator. This constraction is explained in Chapter 3 of [2].

For an operator $T \in \mathcal{B}^{\mathrm{r}}(\mathcal{V})$, the right $S$-resolvent is defined via the formula

$$
S_{R}^{-1}(\mathbf{s}, T)=-\left(T-\mathbf{s}^{\star}\right)\left(T^{2}-2 \Re(\mathbf{s}) T+\|\mathbf{s}\|\right)^{-1}, \mathbf{s} \in \rho_{C l}(T)
$$

(which is the right version of formula (3.5) from [2]; see also formula (4.47) from [2]). Fixing an element $\kappa \in \mathbb{S}_{n}$, and a spectrally saturated open set $\Omega \subset \mathfrak{P}_{n}$, for $\Phi \in \mathcal{R}_{s}\left(\Omega, \mathfrak{C}_{n}\right)$ one sets

$$
\Phi(T)=\frac{1}{2 \pi} \int_{\Sigma_{\kappa}} \Phi(\mathbf{s}) d \mathbf{s}_{\kappa} S_{R}^{-1}(\mathbf{s}, T),
$$

where $\Sigma_{\kappa}$ consists of a finite family of closed curves, piecewise smooth, positively oriented, being the boundary of the set $\Theta_{\kappa}=\{\mathbf{s}=u+v \kappa \in \Theta ; u, v \in \mathbb{R}\}$, where $\Theta \subset \Omega$ is a spectrally saturated open set containing 
$\sigma_{C l}(T)$, and $d \mathbf{s}_{\kappa}=-\kappa d u \wedge d v$. Formula (21) is a slight extension of the (right) functional calculus, as defined in [2], Theorem 3.3.2 (see also formula (4.54) from [2]).

Our Corollary 3 constructs, in particular, an analytic functional calculus with functions from $\mathcal{O}_{s}\left(U, \mathfrak{K}_{n}\right)$, where $U$ is a neighborhood of the complex spectrum of a given Cliffordian operator, leading to another Clifford operator, replacing formally the complex variable with that operator. We can show that those functional calculi are equivalent. This is a consequence of the isomorphism of the spaces $\mathcal{O}_{s}\left(U, \mathfrak{K}_{n}\right)$ and $\mathcal{R}_{s}\left(U_{\sigma}, \mathfrak{C}_{n}\right)$, implied by Theorems 4 and 5 .

Let us give a direct argument concerning the equivalence of those analytic functional calculi. Because the space $V_{\mathbb{C}}$ is also a $\mathrm{Cl}$-space, we may apply these formulas to the extended operator $T_{\mathbb{C}} \in \mathcal{B}^{\mathrm{r}}\left(\mathcal{V}_{\mathbb{C}}\right)$, replacing $T$ by $T_{\mathbb{C}}$ in formulas (20) and (21). In fact, using the properties of the morphism $T \mapsto T_{\mathbb{C}}$ (see beginning of Section 7), we deduce that $S_{R}^{-1}(\mathbf{s}, T)_{\mathbb{C}}=S_{R}^{-1}\left(\mathbf{s}, T_{\mathbb{C}}\right)$.

For the function $\Phi \in \mathcal{R}_{s}\left(\Omega, \mathfrak{C}_{n}\right)$ there exists a function $F \in \mathcal{O}_{s}\left(\Omega, \mathfrak{K}_{n}\right)$ such that $F_{\sigma}=\Phi$, by Theorem 4 . Denoting by $\Gamma_{\kappa}$ the boundary of a Cauchy domain in $\mathbb{C}$ containing the compact set $\cup\left\{\sigma(\mathbf{s}) ; \mathbf{s} \in \overline{\Theta_{\kappa}}\right\}$, we can write

$$
\begin{gathered}
\Phi\left(T_{\mathbb{C}}\right)=\frac{1}{2 \pi} \int_{\Sigma_{\kappa}}\left(\frac{1}{2 \pi i} \int_{\Gamma_{\kappa}} F(\zeta)(\zeta-\mathbf{s})^{-1} d \zeta\right) d \mathbf{s}_{\kappa} S_{R}^{-1}\left(\mathbf{s}, T_{\mathbb{C}}\right)= \\
\frac{1}{2 \pi i} \int_{\Gamma_{\kappa}} F(\zeta)\left(\frac{1}{2 \pi} \int_{\Sigma_{\kappa}}(\zeta-\mathbf{s})^{-1} d \mathbf{s}_{\kappa} S_{R}^{-1}\left(\mathbf{s}, T_{\mathbb{C}}\right)\right) d \zeta
\end{gathered}
$$

It follows from the complex linearity of $S_{R}^{-1}\left(\mathbf{s}, T_{\mathbb{C}}\right)$, and via an argument similar to that for getting formula (4.49) in [2], that

$$
(\zeta-\mathbf{s}) S_{R}^{-1}\left(\mathbf{s}, T_{\mathbb{C}}\right)=S_{R}^{-1}\left(\mathbf{s}, T_{\mathbb{C}}\right)\left(\zeta-T_{\mathbb{C}}\right)-1,
$$

whence

$$
(\zeta-\mathbf{s})^{-1} S_{R}^{-1}\left(\mathbf{s}, T_{\mathbb{C}}\right)=S_{R}^{-1}\left(\mathbf{s}, T_{\mathbb{C}}\right)\left(\zeta-T_{\mathbb{C}}\right)^{-1}+(\zeta-\mathbf{s})^{-1}\left(\zeta-T_{\mathbb{C}}\right)^{-1}
$$

and therefore,

$$
\begin{gathered}
\frac{1}{2 \pi} \int_{\Sigma_{\kappa}}(\zeta-\mathbf{s})^{-1} d \mathbf{s}_{\kappa} S_{R}^{-1}\left(\mathbf{s}, T_{\mathbb{C}}\right)=\frac{1}{2 \pi} \int_{\Sigma_{\kappa}} d \mathbf{s}_{\kappa} S_{R}^{-1}\left(\mathbf{s}, T_{\mathbb{C}}\right)\left(\zeta-T_{\mathbb{C}}\right)^{-1}+ \\
\frac{1}{2 \pi} \int_{\Sigma_{\kappa}}(\zeta-\mathbf{s})^{-1} d \mathbf{s}_{\kappa}\left(\zeta-T_{\mathbb{C}}\right)^{-1}=\left(\zeta-T_{\mathbb{C}}\right)^{-1},
\end{gathered}
$$

because

$$
\frac{1}{2 \pi} \int_{\Sigma_{\kappa}} d \mathbf{s}_{\kappa} S_{R}^{-1}\left(\mathbf{s}, T_{\mathbb{C}}\right)=1 \text { and } \frac{1}{2 \pi} \int_{\Sigma_{\kappa}}(\zeta-\mathbf{s})^{-1} d \mathbf{s}_{\kappa}=0,
$$

as in Theorem 4.8.11 from [2], since the $\mathfrak{K}_{n}$-valued function $\mathbf{s} \mapsto(\zeta-\mathbf{s})^{-1}$ is analytic in a neighborhood of the set $\overline{\Theta_{\kappa}} \subset \mathbb{C}_{\kappa}$ for each $\zeta \in \Gamma_{\kappa}$, respectively. Therefore $\Phi\left(T_{\mathbb{C}}\right)=\Phi(T)_{\mathbb{C}}=F\left(T_{\mathbb{C}}\right)=F(T)_{\mathbb{C}}$, implying $\Phi(T)=F(T)$.

Conversely, choosing a function $F \in \mathcal{O}_{s}\left(\Omega, \mathfrak{K}_{n}\right)$, and denoting by $\Phi \in \mathcal{R}_{s}\left(\Omega, \mathfrak{C}_{n}\right)$ its Cauchy transform, the previous computation in reverse order shows that $\Phi(T)=F(T)$. Consequently, for a fixed $T \in \mathcal{B}^{\mathrm{r}}(\mathcal{V})$ and the maps $\Theta: \mathcal{R}_{s}\left(\Omega, \mathfrak{C}_{n}\right) \mapsto \mathcal{B}^{\mathrm{r}}(\mathcal{V})$, with $\Theta(\Phi)=\Phi(T)$, and $\Psi: \mathcal{O}_{s}\left(\Omega, \mathfrak{K}_{n}\right) \mapsto \mathcal{B}^{\mathrm{r}}(\mathcal{V})$, with $\Psi(F)=F(T)$, we must have the equality $\Psi=\Theta \circ \mathbb{C}[\star]$, where $C\left[{ }^{\star}\right]$ is the Cauchy transform.

Remark 18. Unlike in [2,3], our approach permits to obtain a version of the spectral mapping theorem in a classical stile, via direct arguments. Recalling that $\mathcal{R}_{s, n}(\Omega)$ is the subalgebra of $\mathcal{R}_{s}\left(\Omega, \mathfrak{C}_{n}\right)$ whose elements are also in $\mathcal{J F}\left(\Omega, \mathfrak{C}_{n}\right)$ (see Theorem 4), for every operator $T \in \mathcal{B}^{\mathrm{r}}(\mathcal{V})$ and every function $\Phi \in \mathcal{R}_{s, n}(\Omega)$ one has $\sigma_{C l}(\Phi(T))=\Phi\left(\sigma_{C l}(T)\right)$, via Theorem 3.5.9 from [2]. Using our approach, for every function $f \in \mathcal{O}_{s}(U)$, one has $f\left(\sigma_{\mathbb{C}}(T)\right)=\sigma_{\mathbb{C}}(f(T)$ ), directlly from the corresponding (classical) spectral mapping theorem in [4]. This result is parallel to that from [2] mentioned above, also giving an explanation for the former, via the isomorphism of the spaces $\mathcal{O}_{s}(U)$ and $\mathcal{R}_{s, n}(\Omega)$ 


\section{Application to Tuples of Real Operators}

The special case exhibited at the beginning of Remark 17 is largely treated in [2], in connection with slice regular functions and analytic functional calculus. In this section, we shall briefly present some consequences of the results from the previous ones, valid, in particular, for not necessarily commuting tuples of linear operators acting on a given real Banach space $\mathcal{V}$. We adapt, with our notation, the framework of [2]. For a fixed integer $n \geq 1$, we consider the real vector space $\mathcal{V}_{n}=\mathcal{V} \otimes_{\mathbb{R}} \mathfrak{C}_{n}$. The elements of $\mathcal{V}_{n}$ will be written under the form $\mathbf{v}=\sum_{J} v_{J} e_{J}$, with $v_{J} \in \mathcal{V}$, where $J \prec \mathbb{N}_{n}$, and the symbol " $\otimes$ “ will be omitted. The space $v_{n}$ is a two-sided $\mathfrak{C}_{n}$-module, with the operations

$$
\left(\sum_{J} u_{J} e_{J}\right)\left(\sum_{K} v_{K} e_{K}\right)=\sum_{J, K} u_{J} v_{K} e_{J} e_{K},\left(\sum_{K} v_{K} e_{K}\right)\left(\sum_{J} u_{J} e_{J}\right)=\sum_{K, J} u_{J} v_{K} e_{K} e_{J},
$$

for all elements $\sum_{J} u_{J} e_{J} \in \mathfrak{C}_{n}, \sum_{K} v_{K} e_{K} \in \mathcal{V}_{n}$.

Fixing a norm $\left\|{ }^{\star}\right\|$ on $\mathcal{V}$, we define a norm on $\mathcal{V}_{n}$ by $\|\mathbf{v}\|_{n}=\sum_{J}\left\|v_{J}\right\|$, where $\mathbf{v}=\sum_{J} v_{J} e_{J}$.

Following [2], the space $\mathcal{V}_{n}$ is a Banach $\mathfrak{C}_{n}$-module if there exists a constant $C \geq 1$ such that $\|\mathbf{a v}\|_{n},\|\mathbf{v a}\|_{n}$ are both majored by $C|\mathbf{a}|\|\mathbf{v}\|_{n}$, for all $\mathbf{a} \in \mathfrak{C}_{n}, \mathbf{v} \in \mathcal{V}_{n}$. With our terminology, in this case the space $\mathcal{V}_{n}$ is a Banach $\mathrm{Cl}$-space.

Let $\mathcal{B}(\mathcal{V})$ be the algebra of $\mathbb{R}$-linear operators of the real Banach space $\mathcal{V}$. For a fixed family $\left\{T_{J}\right\}_{J \prec \mathbb{N}_{n}}$, we define an operator $\mathbf{T}=\sum_{J} T_{J} e_{J}$, acting on $\nu_{n}$ via the formula

$$
\mathbf{T}(\mathbf{v})=\sum_{J} \sum_{K} T_{J}\left(v_{K}\right) e_{J} e_{K}, \mathbf{v}=\sum_{K} v_{K} e_{K} \in V_{n} .
$$

The set of all operators of this form will be denoted by $\mathcal{B}_{n}\left(\mathcal{V}_{n}\right)$. Setting the norm $\|\mathbf{T}\|=\sum_{J}\left\|T_{J}\right\|$, the set $\mathcal{B}_{n}\left(\mathcal{V}_{n}\right)$ is a unital real Banach algebra (see [2], page 82).

Note that, with $\mathbf{T} \in \mathcal{B}_{n}\left(\mathcal{V}_{n}\right)$ represented as above, and $\mathbf{v}=\sum_{K} v_{K} e_{K} \in \mathcal{V}_{n}$, we have

$$
\mathbf{T}(\mathbf{v a})=\sum_{J, K, L} a_{L} T_{J}\left(v_{K}\right) e_{J} e_{K} e_{L}=\mathbf{T}(\mathbf{v}) \mathbf{a}, \mathbf{a}=\sum_{L} a_{L} e_{L},
$$

showing that $\mathbf{T} \in \mathcal{B}^{\mathrm{r}}\left(\mathcal{V}_{n}\right)$. In other words, $\mathcal{B}_{n}\left(\mathcal{V}_{n}\right) \subset \mathcal{B}^{\mathrm{r}}\left(\mathcal{V}_{n}\right)$, and the inclusion is strict, as simple examples show. An analytic functional calculus for the operator $\mathbf{T}=\sum_{J} T_{J} e_{J} \in \mathcal{B}^{\mathrm{r}}\left(\mathcal{V}_{n}\right)$ can be obtained directly, as a consequence of Theorem 7. Nevertheless, as in [2], with minor modifications, we may replace the algebra $\mathcal{B}^{\mathrm{r}}\left(\mathcal{V}_{n}\right)$ by the algebra $\mathcal{B}_{n}\left(\mathcal{V}_{n}\right)$.

First of all, we consider the complexification $\mathcal{V}_{n \mathbb{C}}=\mathcal{V}_{n}+i \mathcal{V}_{n}$ of the real vector space $\mathcal{V}_{n}$. If $\mathbf{T}=\sum_{J} T_{J} e_{J} \in$ $\mathcal{B}_{n}\left(\mathcal{V}_{n}\right)$, and $\mathbf{w}=\sum_{K} w_{K} e_{K} \in \mathcal{V}_{n \mathbb{C}}$, with $w_{K}=u_{K}+i v_{K}, u_{K}, v_{K} \in \mathcal{V}_{n}$, then

$$
\mathbf{T}_{\mathbb{C}}(\mathbf{w})=\sum_{J, K}\left(T_{J}\left(u_{K}\right)+i T_{J}\left(v_{K}\right)\right) e_{J} e_{K}=\sum_{J, K} T_{J \mathbb{C}}\left(w_{K}\right) e_{J} e_{K} \in \mathcal{B}_{n}\left(v_{n \mathbb{C}}\right) .
$$

The conjugation $\mathbf{w}=\sum_{K} w_{K} e_{K} \mapsto \overline{\mathbf{w}}=\sum_{K} \bar{w}_{K} e_{K}$ on $\mathcal{V}_{n \mathbb{C}}$, say $C$, induces a conjugation $\mathbf{S} \mapsto \mathbf{S}^{b}$ via the definition $\mathbf{S}^{b}=C \mathbf{S} C$ for all $\mathbf{S} \in \mathcal{B}_{n}\left(\mathcal{V}_{n \mathbb{C}}\right)$. Moreover, $\mathbf{S}=\mathbf{S}^{b}$ if and only if $\mathbf{S}\left(\mathcal{V}_{n}\right) \subset \mathcal{V}_{n}$, and $\mathbf{T}_{\mathbb{C}}^{b}=\mathbf{T}_{\mathbb{C}}$ for all $\mathbf{T} \in \mathcal{B}_{n}\left(\mathcal{V}_{n}\right)$.

As in the case of real operators (see [19]), we define the complex spectrum of the operator $\mathbf{T} \in \mathcal{B}_{n}\left(\mathcal{V}_{n}\right)$ by the equality $\sigma_{\mathbb{C}}(\mathbf{T})=\sigma\left(\left(\mathbf{T}_{\mathbb{C}}\right)\right.$.

Theorem 8. Let $\mathcal{V}$ be a real Banach space, let $\mathbf{T}=\sum_{J} T_{J} e_{J}$ acting on $\mathcal{V}_{n}$, and let $U \subset \mathbb{C}$ be a conjugate symmetric open set with $\sigma_{\mathbb{C}}(\mathbf{T}) \subset U$. Then there exists an assignment

$$
\mathcal{O}_{s}\left(U, \mathcal{B}_{n}\left(\mathcal{V}_{n \mathbb{C}}\right) \ni F \mapsto F(\mathbf{T}) \in \mathcal{B}_{n}\left(\mathcal{V}_{n}\right)\right.
$$

is an $\mathbb{R}$-linear map, and the restricted map

$$
\mathcal{O}_{s}(U) \ni f \mapsto f(\mathbf{T}) \in \mathcal{B}_{n}\left(\mathcal{V}_{n}\right)
$$


is a unital real algebra morphism.

Moreover, the following properties are true:

(1) for all $F \in \mathcal{O}_{s}\left(U, \mathcal{B}_{n}\left(\mathcal{V}_{n \mathbb{C}}\right), f \in \mathcal{O}_{s}(U)\right.$, we have $(F f)(\mathbf{T})=F(\mathbf{T}) f(\mathbf{T})$.

(2) for every polynomial $P(\zeta)=\sum_{j=0}^{m} A_{j} \zeta^{j}, \zeta \in \mathbb{C}$, with $A_{j} \in \mathcal{B}_{n}\left(\mathcal{V}_{n}\right)$ for all $j=0,1, \ldots, m$, we have $P(\mathbf{T})=\sum_{j=0}^{m} A_{j} \mathbf{T}^{j} \in \mathcal{B}_{n}\left(\mathcal{V}_{n}\right)$.

Proof. The proof follows the lines of that of Theorem 7 (see also Theorem 2 from [19]). We only note that a suitable version of the first part of Theorem 6 (or Theorem 1 and Remark 7 from [19]) is necessary, with minor modifications. The detaile are left to the reader.

Remark 19. As in [2], fixing an arbitrary family $\left\{T_{j}\right\}_{j=1}^{n}$, one considers the operator $\mathbf{T}=\sum_{j=1}^{n} T_{j} e_{j}$, which acts on $\mathcal{V}_{n}$ via the formula

$$
\mathbf{T}(\mathbf{v})=\sum_{j=1}^{n} \sum_{K} T_{j}\left(v_{K}\right) e_{j} e_{K}, \mathbf{v}=\sum_{K} v_{K} e_{K} \in \mathcal{V}_{n} .
$$

In the previous statement we may replace the algebra $\mathcal{O}_{s}\left(U, \mathcal{B}_{n}\left(\mathcal{V}_{n \mathbb{C}}\right)\right)$ by the smaller algebra $\mathcal{O}_{s}\left(U, \mathfrak{K}_{n}\right)$, regarding its elements as left multiplication operators (see Corollary 3 ). In this way, we obtain an analytic functional calculus for tuples of real operators, using analytic stem functions.

A parallel version of some results from this section was discussed in the first part of [2], using the slice regular functions to construct functional calculi.

The author is indebted to the referee for a careful reading of the original version of this paper, in particular for pointing out several typos.

Conflict of interest: Author states no conflict of interest.

\section{References}

[1] F. Colombo, I. Sabadini and D. C. Struppa, Slice monogenic functions, Israel J. Math. 171 (2009), 385-403.

[2] F. Colombo, I. Sabadini and D. C. Struppa, Noncommutative Functional Calculus, Theory and Applications of Slice Hyperholomorphic Functions, Progress in Mathematics Vol. 28, Birkhäuser/Springer Basel AG, Basel, 2011.

[3] F. Colombo, J. Gantner, and D. P. Kimsey, Spectral Theory on the S-spectrum for Quaternionic Operators, Birkhäuser, 2018.

[4] N. Dunford and J. T. Schwartz, Linear Operators, Part I: General Theory, Interscience Publishers, New York, 1958. Part III: Spectral Operators, Wiley-Interscience, New York, 1971.

[5] J. Eschmeier and M. Putinar, Spectral Decompositions and Analytic Sheaves, Clarendon Press, Oxford, 1996.

[6] R. Fueter, Über eine Hartogs'schen Satz, Comment. Math. Helv. 12 (1939/40), 75-80.

[7] G. Gentili and D. C. Struppa, A new theory of regular functions of a quaternionic variable, Advances in Mathematics 216 (2007), 279-301.

[8] R. Ghiloni, V. Moretti and A. Perotti, Continuous slice functional calculus in quaternionic Hilbert spaces, Rev. Math. Phys. 25 no.4 (2013), p. 1350006-1-1350006-83. - DOI: 10.1142/S0129055X13500062

[9] G. N. Hile and P. Lounesto, Matrix Representations of Clifford Algebras, Linear Algebra and its Applications, 128 (1990), 51-63.

[10] B. Jefferies, Spectral Properties of Noncommuting Operators, Lecture Notes in Mathematics 1843, Springer-Verlag, Berlin, 2004

[11] G. Laville and I. Ramadanoff, Holomorphic Cliffordian Functions, Adv. Appl. Clifford Algebras, 8 (1998), 323-340.

[12] E. Nelson, Operants: A functional calculus for non-commuting operators, in: Functional analysis and related fields, Proceedings of a conference in honour of Professor Marshal Stone, Univ. of Chicago, May 1968, Springer-Verlag, Berlin/Heidelberg/New York, 1970, pp. 172-187.

[13] I. R. Porteous, Topological Geometry, Van Nostrand Reinhold Company, New York-Toronto-Melbourne, 1969

[14] J. L. Taylor, The analytic-functional calculus for several commuting operators, Acta Math. 125 (1970), 1-38.

[15] J. L. Taylor, A general framework for a multi-operator functional calculus. Advances in Math. 9 (1972), 183-252.

[16] J. L. Taylor, Functions of several noncommuting variables, Bull. Amer. Math. Soc. 79 (1973), 1-34.

[17] F.-H. Vasilescu, Analytic functional calculus and spectral decompositions, D. Reidel Publishing Co., Dordrecht and Editura Academiei R. S. R., Bucharest, 1982.

[18] F.-H. Vasilescu, Quaternionic Regularity via Analytic Functional Calculus. Integr. Equ. Oper. Theory 92, 18 (2020). https://doi.org/10.1007/s00020-020-2574-7. 
[19] F.-H. Vasilescu, Spectrum and Analytic Functional Calculus in Real and Quaternionic Frameworks, Pure and Applied Functional Analysis (to appear); see also arXiv:2003.05263.v2 